\title{
Les polymères comme matériaux désordonnés : l'apport de la diffusion de neutrons
}

\author{
F. Boué
}

Laboratoire Léon Brillouin, CNRS/CEA, CE-Saclay, 91191 Gif-sur-Yvette cedex, France

\begin{abstract}
Résumé : Les polymères à l'état amorphe, liquide ou vitreux forment des matériaux désordonnés aux propriétés spécifiques. Les larges gammes de distance impliquées, locale, semi-locale, intermédiaire, globale, de 1 à $500 \mathrm{~nm}$ font de la Diffusion de Neutrons aux Petits Angles (DNPA ${ }^{\circ}$, mais aussi de la diffusion inélastique par temps de vol et écho de spin, et de la réflectivité des outils d'étude privilégiés. Nous détaillerons le cas de la châne gaussienne dans le fondu, sa statique et sa dynamique à l'état libre puis le cas de la chaîne vermiforme. Nous considérerons ensuite les solutions en bon solvant, en statique et dynamique, les solutions de polyélectrolytes, l'enchevêtrement et la reptation, les réseaux et gels de chaînes réticulées. Ce qui nous amènera à des exemples de diffusion par des objets déformés, ou sous écoulement (rhéo-DNPA). Nous décrirons brièvement le cas de systèmes mixtes et les avantages de la variation de contraste, ainsi que l'étude des interfaces par DNPA ou par réflectivité.
\end{abstract}

\section{PRESENTATION: LES POLYMERES. UN MATERIAU DESORDONNE IMPLIQUANT DE LARGES ECHELLES}

\subsection{Polymères : importance pratique, industrielle}

11 est évident pour tous que les polymères ont une grande importance en tant que matériaux, structuraux ou à haute valeur ajoutée, dans la vie quotidienne (construction, transport, sport) ou dans les applications biologiques et médicales, en masse - c'est-à-dire sans solvant, comme dans les « matières plastiques", ou en solution, dans l'eau en particulier.

Les polymères sont constitués de séquences d'éléments chimiquement identiques (Figure 1). En masse ils peuvent, lorsque leur enchaînement est suffisamment régulier (isotactique, ou syndiotactique), former des zones cristallisées coexistant avec des zones amorphes (à cause des réarrangements complexes des chaînes impliqués dans le processus de cristallisation) dans une structure semi- cristalline. Cela concerne de nombreux matériaux industriels, mais nous ne considérerons ici que le cas amorphe, illustré par le cas simple des non stéréoréguliers.

\subsection{Polymères amorphes : un matériau désordonaé}

Les polymères amorphes sont un exemple particulier de matériaux désordonnés: ils présentent un état liquide et un étaî vitreux selon la température, avec dans les deux cas une structure identique à celle des liquides ou des verres moléculaires, d'un certain point de vue hé à l'échelle locale essentiellement. D'un autre point de vue, lié à des tailles plus grandes que les polymères impliquent, les chaînes présentent une conformation et arrangement 
réciproque original. De ce point de vue les polymères sont un matériau d'étude de choix pour la DNPA $[1,2,4]$. Dans un liquide les fluctuations de densité (représentées en Figure 1) dans l'espace direct (abscisse r) sont importantes aux petites échelles, puis se réduisent à des échelles plus grandes à ne traduire que la compressibilité du système. Aux grandes distances peuvent apparaître à nouveau de grandes fluctuations, dues par exemple à des vides. Mais grâce au marquage de grandes molécules comme les chaînes polymères, on peut observer la conformation moyenne d'une chaîne, difficile à atteindre par une autre technique. La Figure montre aussi (encadré supérieur) comment apparaît la chaîne en fonction de l'échelle d'observation.
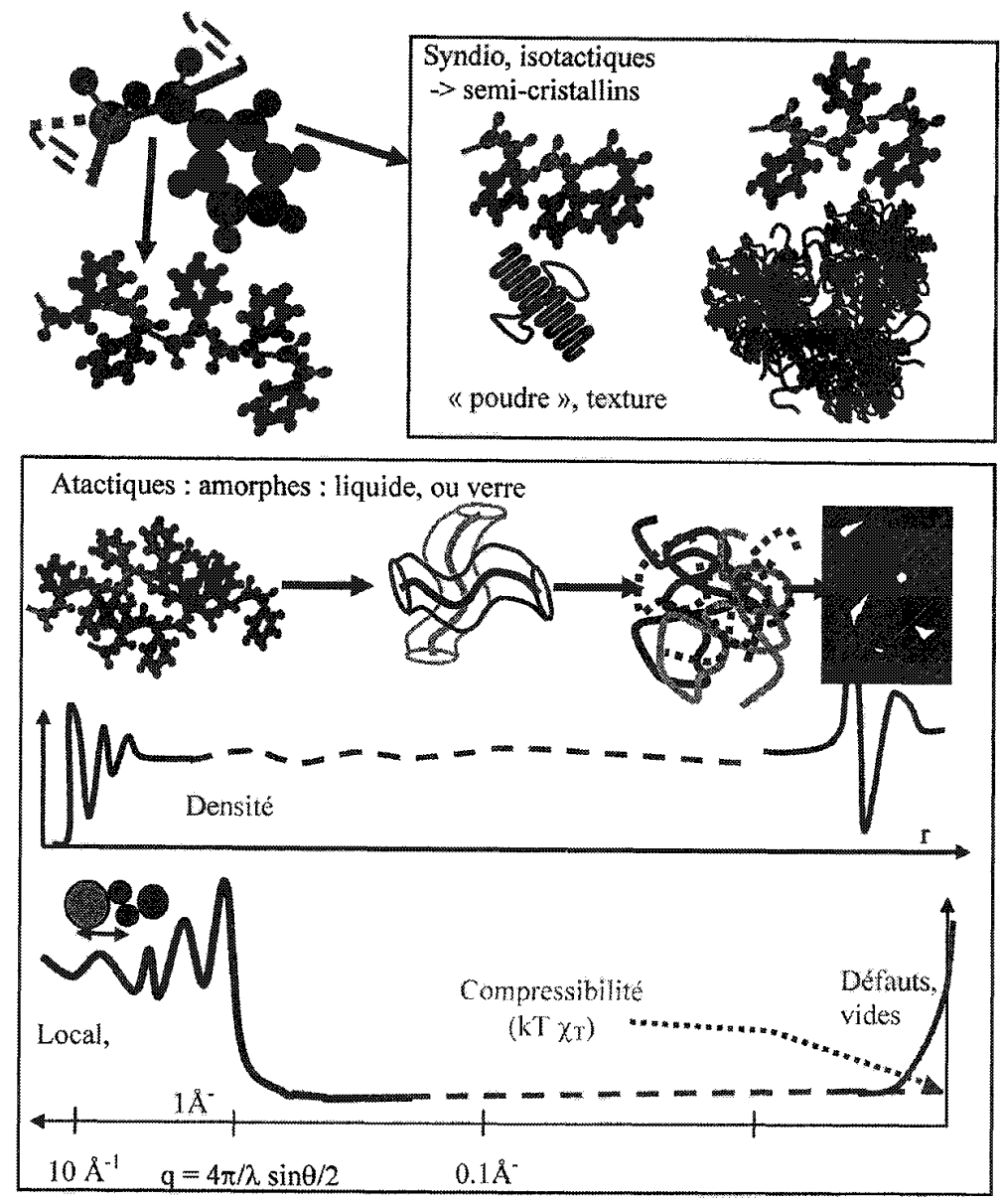

Figure 1 : polymère, état amorphe et corrélations 


\subsection{Exemple d'utilisation de la DNPA pour les polymères : mesure du facteur de forme}

Pour prendre tout de suite un exemple, considérons l'expérience phare pour l'application de la DNPA aux polymères, qui utilise un mélange de chaînes deutériées et non deutériées (supposées identiques à tout autre point de vue : chimique, mécanique, etc...). Pour les neutrons ( $\mathrm{a}$ la différence des rayons $\mathrm{X}$ pour lesquels ce serait un milieu homogène qui ne diffuse pas) ce mélange est un cas particulier de mélange binaire (Figure 2). Le calcul est donné au sein de la Figure 2a. Si on définit le nombre de diffuseur $\mathrm{j}$ par unité de volume, $n_{3}(\mathrm{r})$, l'intensité peut s'écrire comme une somme d'interférences entre deux ondes provenant de deux diffuseurs de longueur de diffusion différentes, $b_{1}$ et $b_{2}$, ce qui se réécrit en trois termes. Or si aux grandes échelles, la densité est constante (pas de vides), les $n_{j}(r)$ sont liés via les volumes molaires $V_{j}$. I est donc décrit par les fluctuations de concentration d'un des deux types des molécules au sein de l'échantillon. Si de plus les deux objets sont identiques à part leur valeur de b (cas d'un marquage), ils sont mélangés au hasard, et l'on observe donc aucune corrélation hormis le long d'une même chaîne : on observe donc le facteur de forme moyen d'une chaîne, tout comme on observerait celui de cubes mélangés au hasard présentés au bas de la Figure $2 b$.

Le préfacteur du signal intra chaîne ainsi obtenu est $\left(n_{1} / v_{1}-n_{2} / v_{2}\right)^{2}$, et est appelé contraste de l'espèce 1 par rapport à l'espèce 2 . L'échelle de $n_{j} / v_{j}$ donnée en Figure $2 b$ permet de comprendre le principe des stratégies de marquage $[1,4]$. En fait on a défini $\Sigma b_{j} / \Sigma v_{j}$ (axe plus à gauche) non pour chaque atome (voir les valeurs des $b_{j}$ pour les noyaux sur l'axe le plus à droite), mais pour un monomère comme la somme des $b_{j}$ de chacun de ses atomes, comme on le ferait pour une petite molécule. Cela revient à considérer que les détails de la structure du monomère sont à une échelle petite pour la DNPA. Dans ces conditions les $b / V$ se regroupent en deux groupes extrêmes, ceux des monomères et molécules de solvant non deutériés (contenant seulement des protons) et à l'autre extrême de l'échelle les solvants et monomères deutériés (seulement des deutérons). Au milieu de l'échelle se trouvent d'autres espèces, comme la silice, ou les protéines.

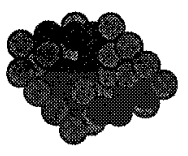

$$
\begin{aligned}
& I=S_{i} S_{k} b_{i} \exp \left(i q r_{i}\right) \cdot b_{k} \exp \left(-i q r_{k}\right) \\
& =S_{i} S_{k} b_{i} n_{i}(r) b_{k} r_{k}\left(r^{\prime}\right) \exp (i q r) \cdot \exp \left(-i q r^{\prime}\right) \\
& =S_{i} S_{k} b_{i} n_{i}(q) b_{k} n_{k}(-q) \\
& =b_{1}^{2} n_{1} n_{1}+2 b_{1} b_{2} n_{1} n_{2}+b_{2}^{2} n_{2} n_{2}
\end{aligned}
$$

Expression de l'incompressibilité :

$$
\begin{aligned}
& \quad V_{1} n_{1}(r)+V_{2} n_{2}(r)=c s t e \Rightarrow V_{1} n_{1}+V_{2} n_{2}=\delta(q)=0 ! ! \\
& I=\left(b_{1}^{2}-2 b_{1} b_{2} V_{1} / V_{2}+\left(b_{2} V_{1} / V_{2}{ }^{2}\right) n_{1} n_{1}\right. \\
& =\left(b_{1}-b_{1} V_{1} / V_{2}\right)^{2} n_{1}(q) n_{1}(-q)
\end{aligned}
$$




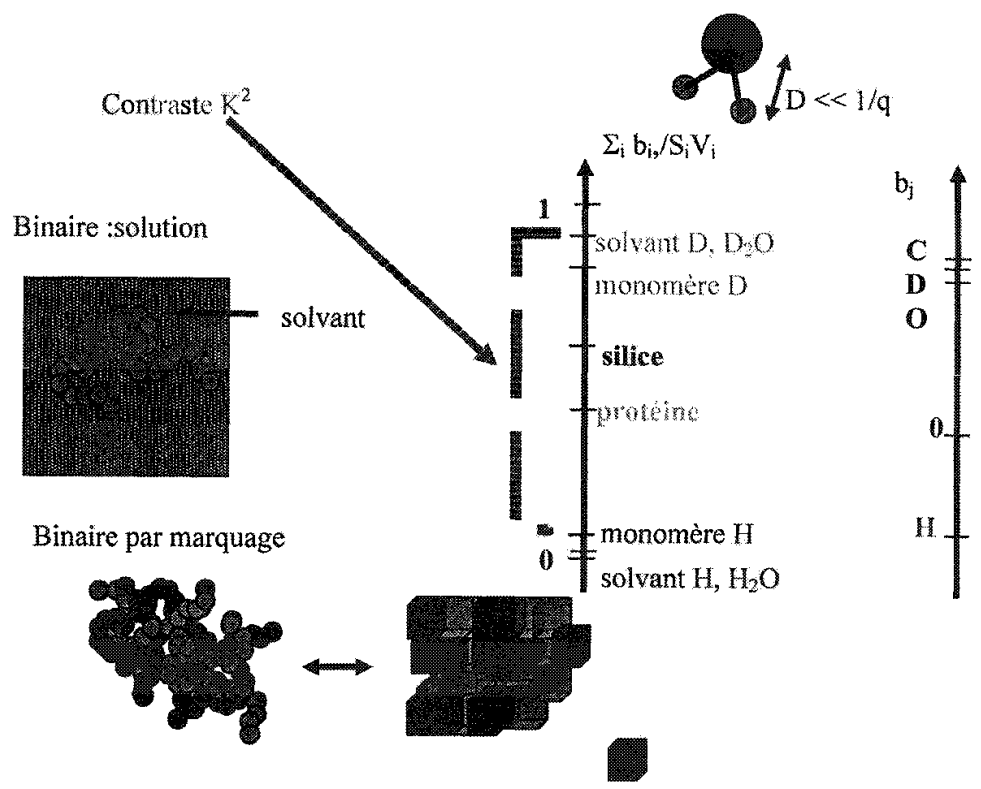

Figure 2b : mélange binaire : échelle de contraste

\subsection{Schéma d'un spectromètre DNPA}

Rappelons à ce stade « l'allure 》 de spectromètres « aux petiss angles » (Figure 3). Comme leur nom l'indique, les petites valeurs de vecteur de diffraction q (de module $4 \pi / \lambda \sin (\theta / 2)$ ) sont obtenues grâce à des angles $\theta$ petits dus à des cellules de détection très petites ( e.g. 0.5 $\mathrm{cm}$ ) situées à de grandes distances (e.g. 10 mètres), pour des longueurs d'onde $\lambda$ grandes ( 2 ঐ̀ 20 Angströms, sélectionnées par un sélecteur mécanique). Cette géométrie longiligne est tout à fait différente de celle utilisée pour les mesures aux grands angles qui caractérisent l'ordre local des matériaux désordonnés. On accède à l'ordre local aux grands angles comme pour les cristaux, mais sans réflexion de Bragg proprement dite. 

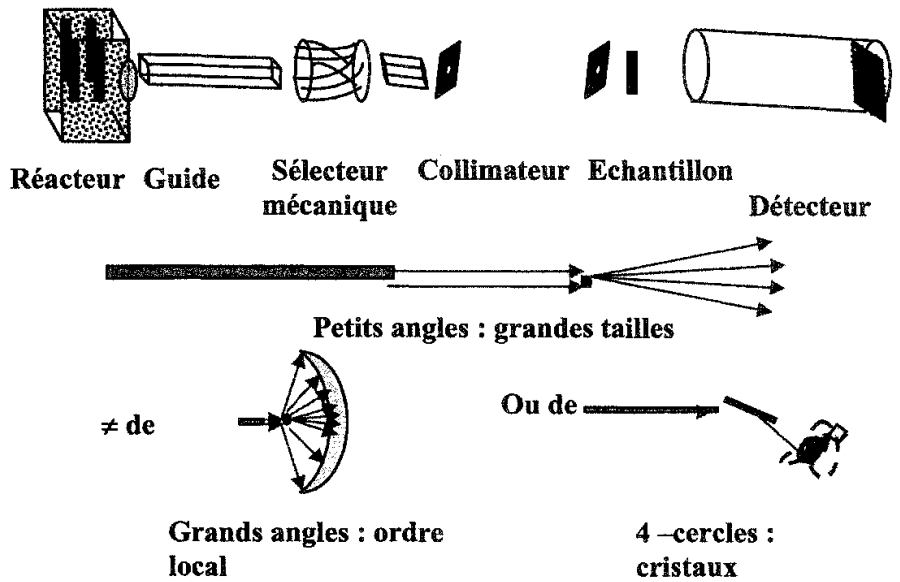

Figure 3 : géométries comparées d'un spectromètre aux «petits angles » et d'un spectromètre aux "grands angles », utilisé pour l'étude de l'ordre local ou de l'ordre cristallin.

\section{DU LOCAL AU SEMI-LOCAL}

Une des premières spécificités des polymères est la large gamme de tailles qu'ils impliquent, avec des structures différentes à ces différentes échelles (Figure 4). Au-dessus des tailles due l'unité répétitive (grands angles, notés GA ), on va ainsi considérer la châne comme un fil caractérisé par son diamètre axial $\mathrm{D}$, et par sa rigidité, via une longueur de persistance $\mathrm{I}_{\mathrm{p}}$. C'est l'échelle semi-locale. La DNPA permet d'accéder à D via des marquages partiels, comme indiqué pour une unité de polystyrène dont seul le noyau benzénique attaché au squelette, est deutérié. La longueur $\mathfrak{l}_{\mathrm{p}}$ induit dans l'intensité de DNPA une variation en $1 / \mathrm{q}$ aux grands qs (petites distances), dont le préfacteur est proportionnel à la densité linéaire de la chaîne $\left(\sim 1 / h_{p}\right)$. Aux plus petits qs (grandes distances), au-delà de $q \sim 1 / h_{p}$, il y a à nouveau un changement de comportement ( cross-over») vers une conformation traduisant un cheminement au hasard, ou bien auto-évitant lorsque la chaîne préfère s'entourer de solvant $\left(1 / \mathrm{q}^{2}\right.$ ou $\left.1 / \mathrm{q}^{5 / 3}\right)$.

Ces différents comportements statiques se retrouvent dans la dynamique d'agitation brownienne du système. Au niveau local, cette dynamique est rapide et pourra être étudiée par spectrométrie temps de vol. Grâce à la présence de nombreux protons, on mesure une diffusion incohérente. On y observera - comme pour d'autres matériaux désordonnés, d'une part des mouvements diffusifs de rotation, au voisinage de la fréquence nulle (pour l'élargissement quasiélastique de variation Lorentzienne en $\left(1 / 1+\Gamma \cdot \omega^{2}\right)$ ), et d'autre part des vibrations locales (" phonons") de type " manivelle» par exemple. La dynamique évoluera, aux plus petits qqs et donc aux plus grands temps, vers des mouvements cohérents, collectifs, du squelette. 


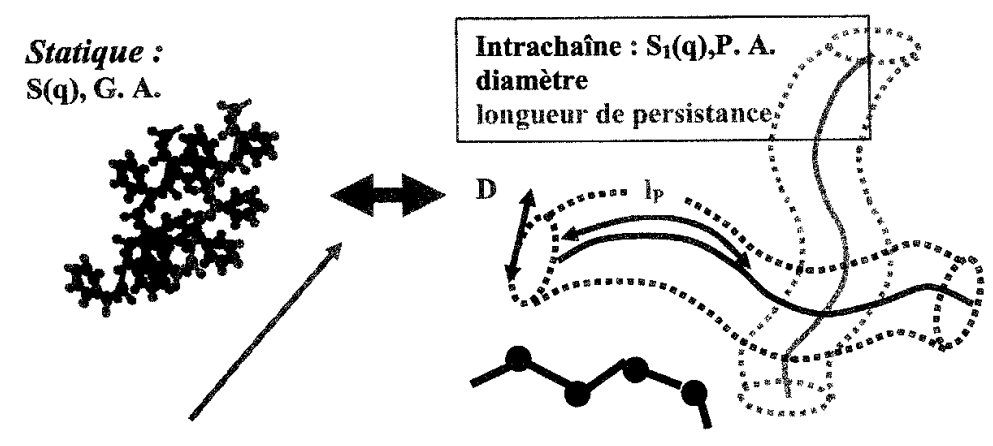

Relation?

$\gg$ simulations

$q<1 / h_{\mathrm{F},}, 1 / q \cdot \exp \left(-q^{2} D^{2}\right)$, cylindre

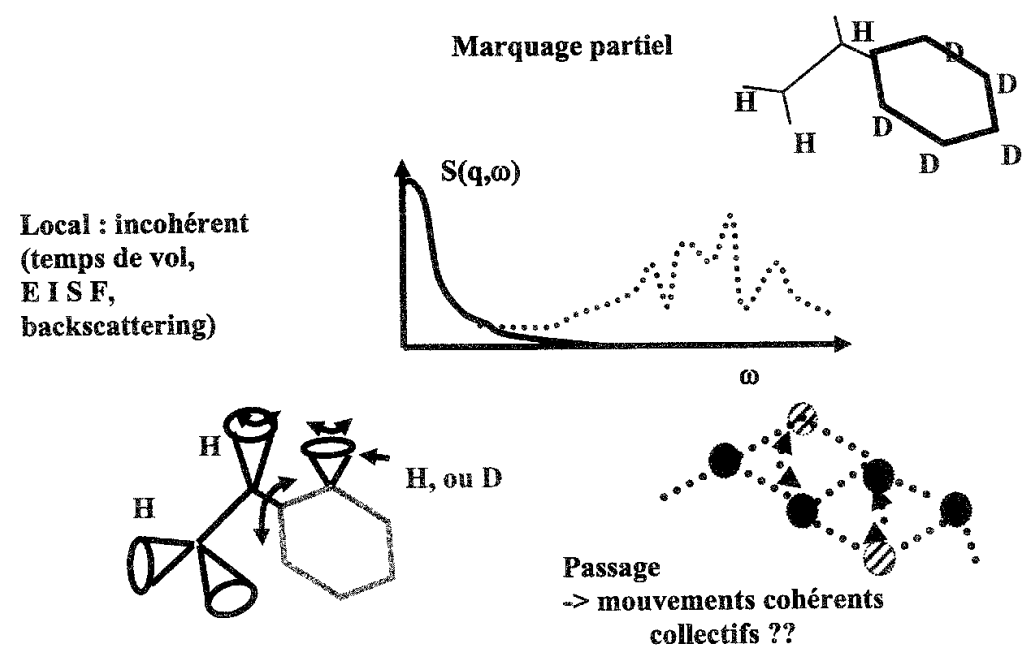

Figure 4 : diffusion élastique et inélastique à l'échelle locale et semi-locale 


\subsection{Dynamique locale et semi-locale, cohérente et incohérente}

En dehors de l'aspect fenêtre de temps des différentes techniques, le fait qu'elles mesurent une diffusion incohérente (temps de vol, mais possible par echo de spin aux temps plus longs) les rend sensibles aux mouvements individuels des protons, alors que la diffusion cohérente (écho de spin), devrait être plus sensible aux mouvements cohérents [1]. La technique d'écho de spin, applicable tant aux petits angles qu'aux grands angles.

Cependant la diffusion cohérente sera également sensible aux fluctuations de densité, quand elles existent. C'est ce que montrent Arbe et al [6] dans la courbe de la Figure 5 (à gauche), représentant la variation du temps caractéristique en fonction du vecteur q. L'incohérent (courbe du haut) se manifeste par une décroissance monotone du temps, alors que le cohérent montre un maximum aux environs du maximum statique correspondant aux distances entre portion de chaînes dans le polymère amorphe (en termes rapides, premier pic de structure dans un liquide).
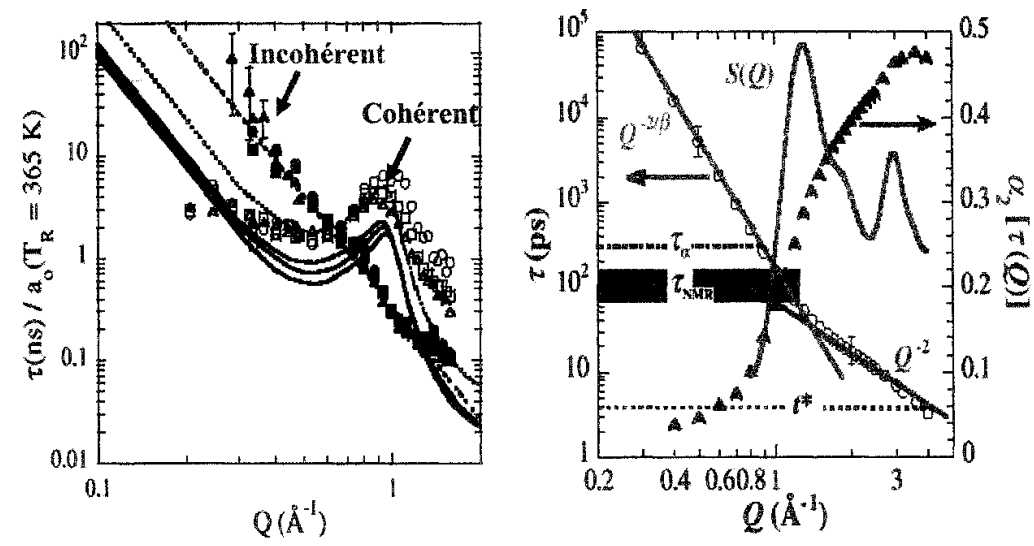

$$
\begin{aligned}
& \text { Crossover }-2 / \beta \rightarrow-2, \\
& \text { «Gaussien } »-\rightarrow>\text { « hétérogène » } \\
& \tau \sim q^{2 / \beta}, \beta=0.5 \equiv \tau \sim q^{-4} \text { Rouse (hasard ?) }
\end{aligned}
$$

Figure 5 : dynamique cohérente et incohérente pour une chaîne de polymère. Comparaison des comportements. D'après B. Farago, A. Arbe, J. Colmenero, R. Faust, U. Buchenau, et D. Richter [6].

L'incohérent reste sensible à la transition vitreuse (donc à des mouvements collectifs) comme le montre la figure de droite, où apparaît pour la variation d'un temps caractéristique $\tau(q)$ (carrés creux rouges) un « cross-over » entre $q^{-2}$ aux grands $q s$ et $q^{-2 / \beta}$ aux petits $q s$. Cette demière peut être analysée en termes de transition vitreuse. Les polymères amorphes présentent en effet une forte transition vitreuse, à cause de leur connectivité jusqu'à des grandes échelles. A priori le système semble plus complexe que des liquides moléculaires. 
Mais le fait qu'une partie des liaisons soit de nature covalente (liaisons $\sigma$ le long du squelette), et donc peu sensibles à la température mène à un comportement de verre dit « fort » dans la classification d'Angell, plus simple à étudier pour tenter de comprendre cette transition que les verres «fragiles", où les liaisons responsables du blocage sont elles mêmes sensibles à la température. La valeur de $\beta$ obtenue ici est de 2 , soit une pente de -4 . Surprenamment cette valeur est aussi celle observée pour une dynamique de Rouse, détaillée plus loin.

Si l'on souhaite observer la diffusion cohérente à plus petit $q$, c'est-à-dire au delà de l'échelle semi-locale, il faut bien sûr à nouveau pratiquer le marquage d'une partie des chaînes. Une telle mesure a été faite par écho de spin pour des chaînes en solution, et sera détaillée plus loin (Paragraphe 2.3.2, Figure 10); cette Figure 10 montre le passage du local au semi-local. La variation de $S(q, t)$ est ajustée à exp.(- $\Gamma . t)$ en temps. Aux grands $q, \Gamma \sim q^{2}$ (mouvements élastiques du squelette analysés selon un modèle d'Akcasu) mais devient proportionnel à $\mathrm{q}^{4}$ à plus petits qs, ce qui correspond à la dynamique de Rouse, comme détaillé au paragraphe 2.3 .

Mais revenons maintenant à la statique pour les plus grandes échelles.

\subsection{Statique : chaîne gaussienne et chaîne vermiforme}

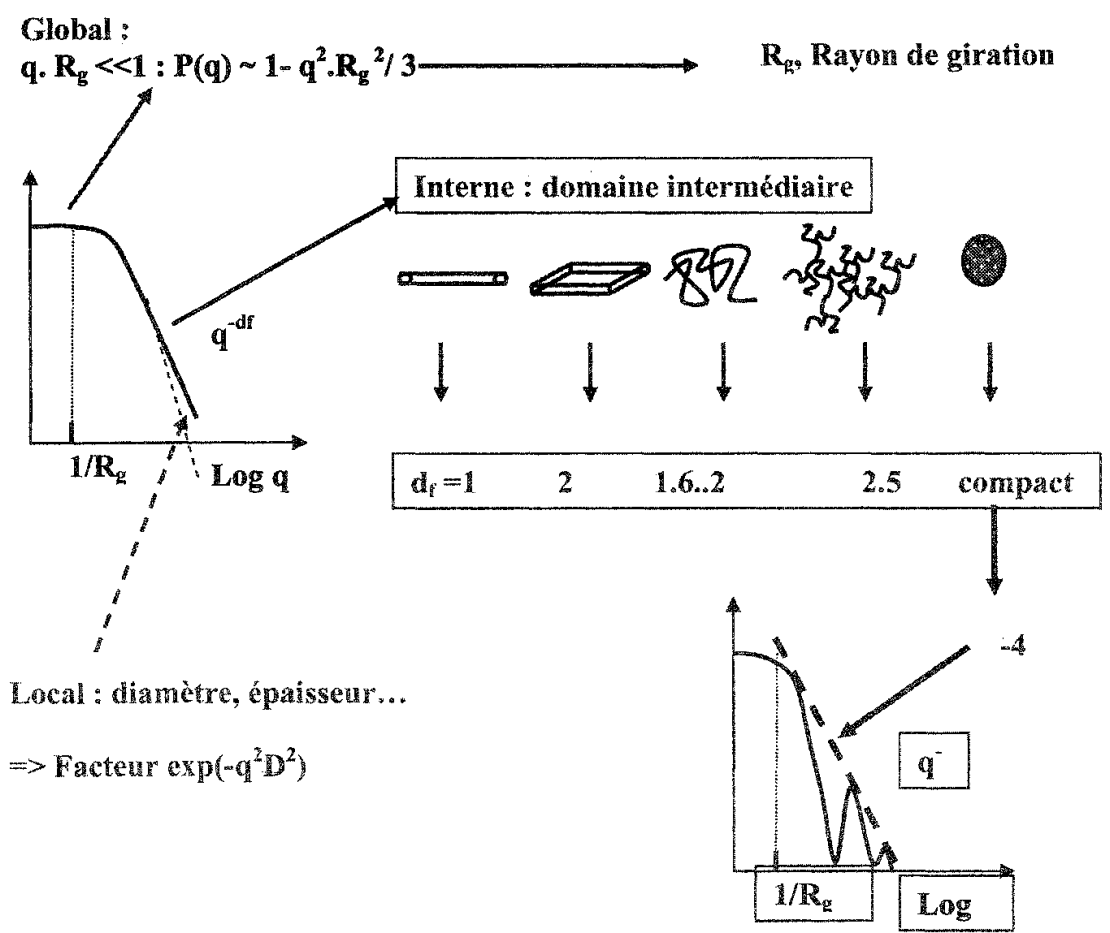

Figure 6 : facteur de forme d'un objet fractal de dimension $d_{f g}$ ou d'un objet compact $\left(\ll d_{f} »=3\right)$, en représentation logarithnique). 


\subsubsection{Comportements pour différents domaines de $q$.}

En masse, à l'état liquide ou vitreux, on peut mesurer le facteur de forme de la chaîne sur l'ensemble des échelles de la chaîne, jusqu'à sa taille globale, caractérisée par le rayon de giration $R_{g}[1,2,4]$. C'est ce que montre la courbe $\log 1 / \log q$ de la Figure 6 pour un fractal de dimension $\mathrm{d}_{\mathrm{f}}$ donnée; cette courbe se compose de trois régions. Aux grands qs, comme déjà évoqué la variation est en $1 / q$ multipliée éventuellement par un préfacteur exp. $\left(-q^{2} \mathrm{D}^{2}\right)$. Pour une chaine, D sera le rayon axial; puis, aux qs moyens, la variation est en $1 / q^{d f}$, sauf si $d_{f}=3$, auquel cas seule la surface de $1^{\prime}$ objet diffuse, ce qui mène à une loi de Porod en $1 / q^{4}$. Enfin aux plus petits qs, le signal tend quand $q->0$, vers une valeur proportionnelle à la masse de l'objet, en l'occurrence la chaîne. Dans cette zone, le facteur de forme $P(q)$ varie (pour tout objet) comme $1-\mathrm{q}^{2} \mathrm{R}_{\mathrm{g}}{ }^{2} / 3$.

Pour une chaîne gaussienne, on retrouve les mêmes comportements avec $d_{f}=2$ (Figure 7). Cela correspond à la transformée de Fourier des fluctuations de concentration d'une seule chaîne cheminant au hasard (entre d'autres termes, à la dimension fractale 2 de la diffusion brownienne). La chaîne chemine au hasard parce qu'elle n'est pas sensible à la différence entre des interactions avec ses propres unités répétitives et des interactions avec celles d'autres chaînes. L'ensemble de ces variations peut se représenter d'une autre façon en représentation dite de Kratky, $q^{2} S_{1}(q)$ versus $q\left(S_{1}(q) \sim M\right.$. $P(q)$ pour une chaîne de masse $\left.M\right)$. La Figure 7 représente une mesure pour une chaîne de polyélectrolyte en solution semi-diluée. On observe clairement la transition $\mathrm{q}^{-2} / \mathrm{q}^{-1}$, qui est correctement ajustée par le modèle dit de la chaîne vermiforme, dont le paramètre essentiel est la longueur de persistance $l_{p}$.

\subsubsection{Longueur de persistance : valeur intrinsèque et effet électrostatique : polyélectrolytes}

Dans le cas de chaînes chargées en solution (polyélectrolytes), les répulsions entre monomères étirent la chaîne. La longueur de persistance est augmentée d'une contribution électrostatique, que l'on peut mesurer en fonction de la concentration $C_{p}$ en chaînes (donc en charges liées au monomère et en contre-ions libres de charge opposée) et en sel ajouté ( $\left.\mathrm{C}_{\mathrm{S}}\right)$ [5]. On observe que le paramètre dominant est la force ionique globale de la solution, due aux ions mobiles (contre-ions de signe opposé à la chaîne et co-ions monovalents du sel ajouté), donc proportionnelle à $2 \mathrm{C}_{\mathrm{S}}+\mathrm{C}_{\mathrm{p}}$ (Figure $8 \mathrm{a}$ ) ; il semble donc que les effets de piégeage des contre-ions dans le puits de potentiel créé par la chaine (effets dits de condensation, qui diminuent la concentration de contre-ions libres de $C_{S}$ à $\left.f_{0} C_{S}, f<1\right)$ ne jouent pas à l'échelle de $L_{p}$ (cf. schémas de la Figure 8b). 


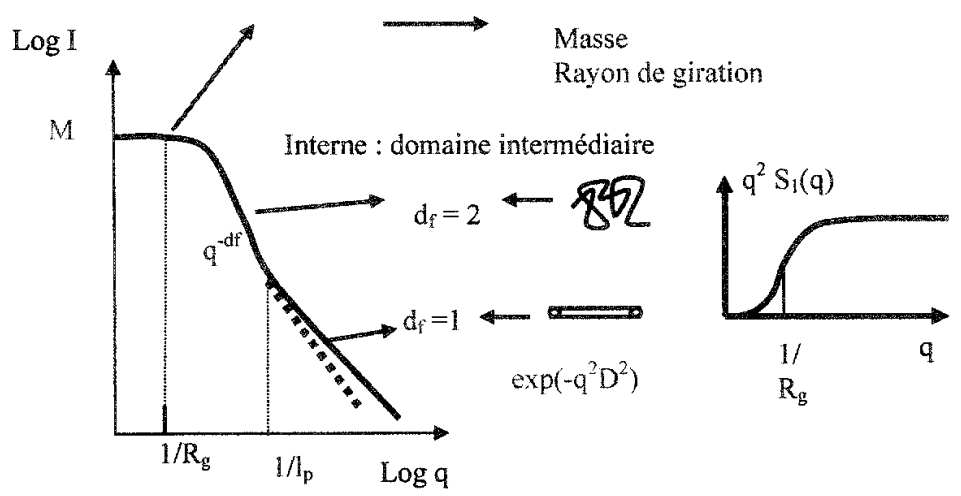

Fit de toute la courbe: chaîne vermiforme

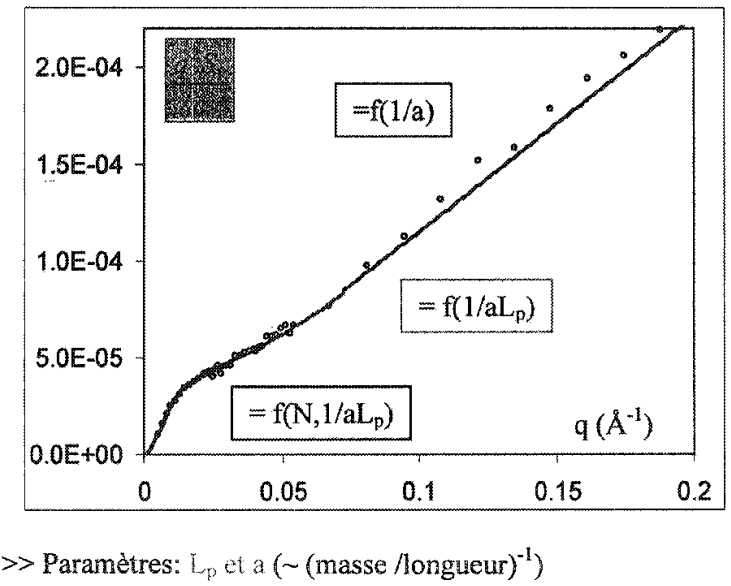

Figure 7: facteur de forme d'une chaine vermiforme (représentation logarithmique et de Kratky) pour trois domaines de q (données de la ref. [5]) 


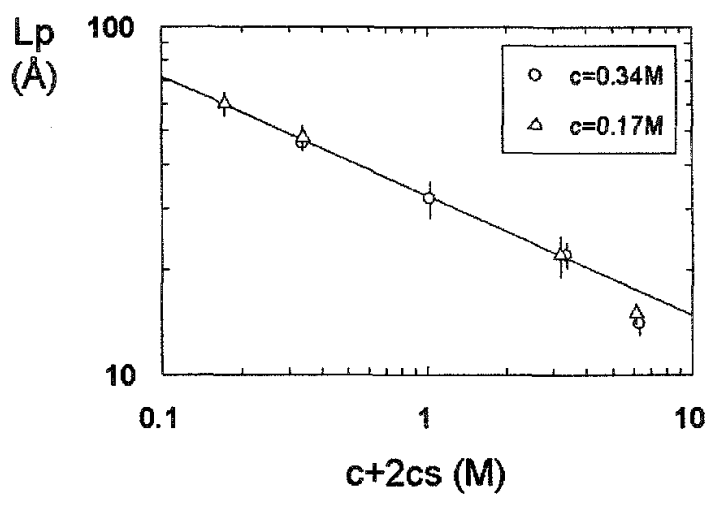

Figure 8a : variation de la longueur de persistance d'une chaîne polyélectrolyte [5] en fonction de la concentration en contre-ions, $C_{p}$ et en ions du sel ajouté, $2 C_{s}$. Cela suggère que les effets de condensation ne sont pas pertinents à cette échelle semi-locale (schémas ci-dessous).

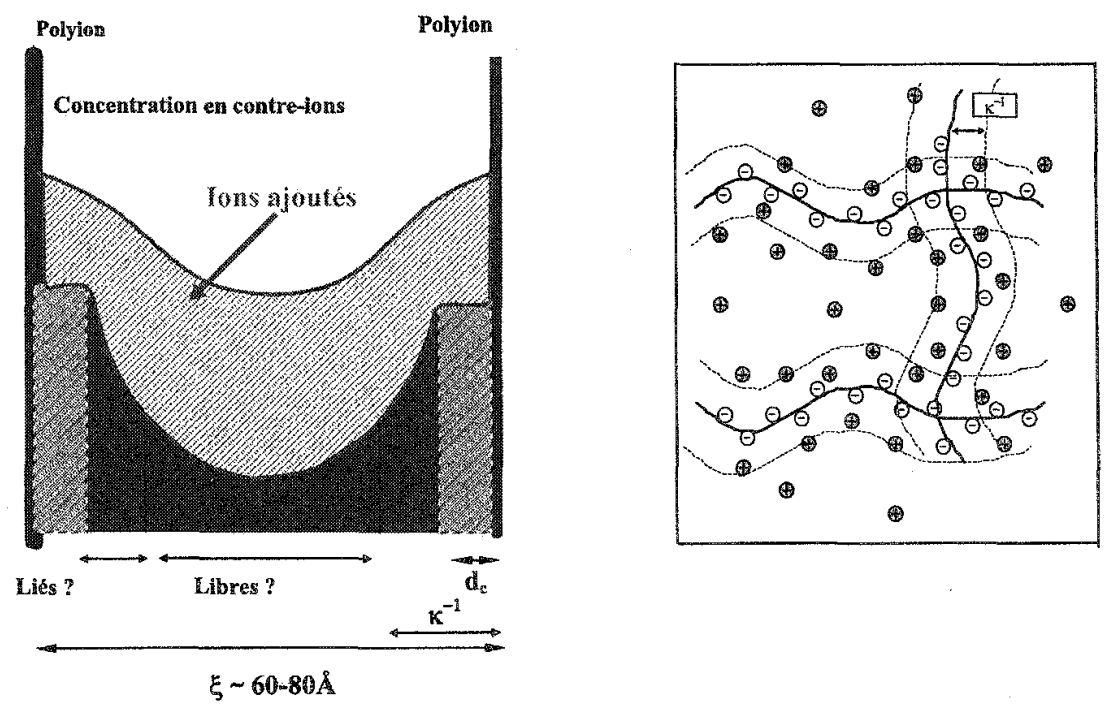

Figure $8 \mathrm{~b}$ : schémas de la distribution spatiale des contre-ions dans une solution semi-diluée de polyélectrolytes. A droite carte des charges moins (sur les chaînes) et plus (contre-ions condensés et libres); à gauche variation de la concentration en ions libres avec $r$, sans ou avec sel ajouté. Dans une cellule élémentaire de la solution, de taille $\xi$ 


\subsection{Dynamique de chaîne gaussienne : modèle de Rouse}

\subsubsection{Modèle}

Connaissant la statique d'une chaîne gaussienne (observée en l'absence d'interactions entre unités statistiques), on peut alors décrire la dynamique de cette chaîne sous l'effet de l'agitation thermique [3]. En plus de forces aléatoires de Langevin, s'exercent sur chaque unité statistique une force de rappel due au ressort entropique que constitue la chaîne Gaussienne (voir équation dans la Figure 9). Tout se passe comme une diffusion Brownienne le long de la chaîne qui est elle-même un chemin au hasard. Finalement la distance moyenne parcourue en un temps $t$ est $\sqrt{ }\left\langle r^{2}\right\rangle \sim t^{1 / 4}$, ce qui est très lent ! c'est sur un «blob» de cette taille que la chaîne renouvelle sa configuration en un temps $t$ (cf. schémas du bas de la Figure 9).

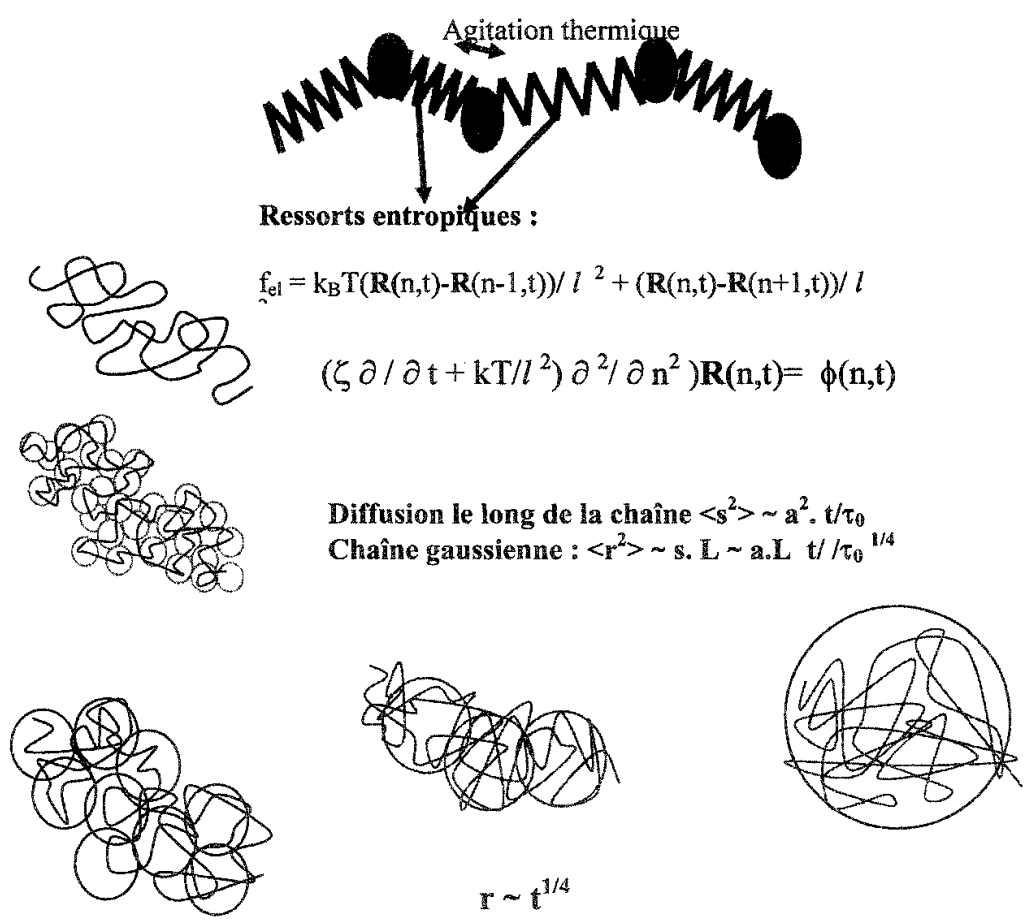

Figure 9 : dynamique de Rouse pour une chaine Gaussienne libre 


\subsubsection{Test du modèle de Rouse}

Le modelle de Rouse a été testé la première fois par diffusion inélastique (temps de vol) par une équipe anglaise (Allen, Higgins, cf. [1]). Il est en fait observable sur une plus grande gamme de $q$ et de $t$ par écho de spin. Si l'on ajuste $S(q, t)$ à exp. $(-\Gamma . t)$ - approximation du premier cumulant, on peut vérifier que $\Gamma$ varie comme $\mathrm{q}^{4}$ (aux grands $\mathrm{q}, \Gamma \sim \mathrm{q}^{2}$, attribué aux mouvements élastiques du squelette analysés par Akcasu, comme déjà dit au dessus).Mais un test plus élégant est possible : la fonction $S(q, t)$ est alors une fonction de $\mathrm{qt}^{1 / 4}$ uniquement, on peut donc tester une superposition des courbes mesurées à différents qs, comme le montre la Figure 10,

TEMPS DE VOL
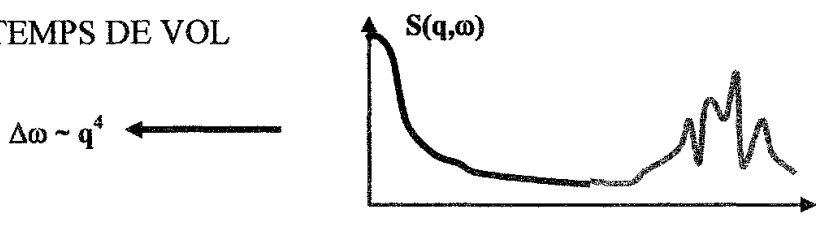

$\mathbf{\omega}$

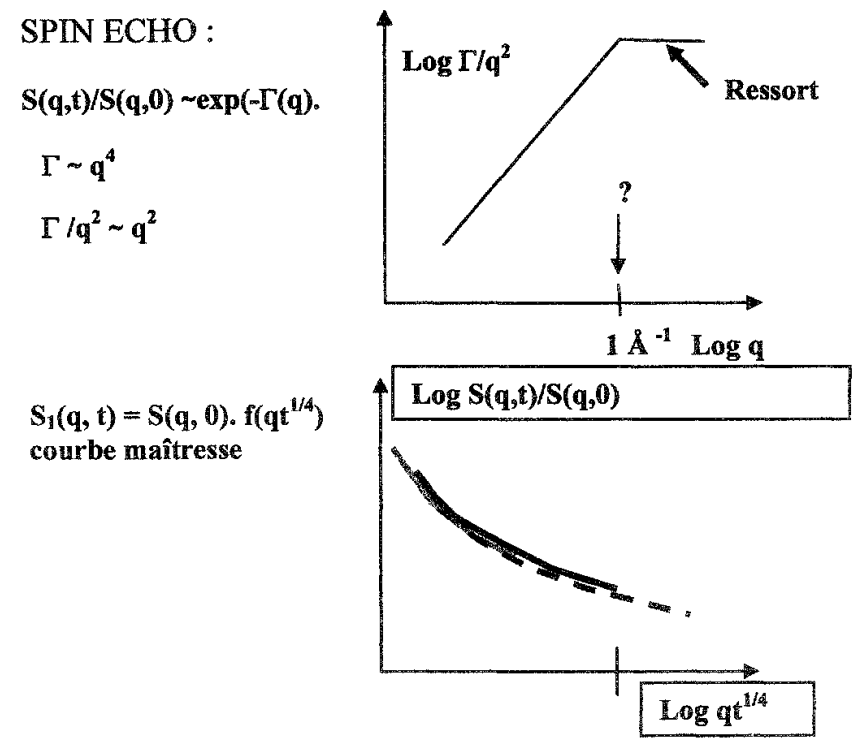

Figure 10 : Tests du modèle de Rouse pour une chaâne marqquée dans un fondu de polymère. 


\section{SOLUTIONS, INTERPENETRATION}

\subsection{Du dilué au semi-dilué}

La physique statistique des solutions de polymères mène également à une organisation et des concepts spécifiques [2]. Tout d'abord, dans le cas d'une solution diluée, au concept de chemin auto-évitant [2]. L'intensité, proportionnelle au facteur de forme, présente une région de Guinier $\left(1-q^{2} R_{g}{ }^{2} / 3\right)$ aux petits $q s$, puis décrôit comme $q^{-3 / 5}$ aux plus grands qs ; l'exposant $3 / 5$ est caractéristique d'un effet d'évitement entre deux portions de chemin aléatoire, les chaînes préférant s'entourer de solvant.

Si l'on augmente la concentration, chaque chaîne ne peut plus occuper seule le volume correspondant au chemin auto-évitant. Le "partage » avec les autres chaînes se fait de telle sorte qu'à petite échelle une portion de chaîne reste seule au sein d'un blob [2] où elle conserve sa conformation de chaîne diluée, tandis qu'à grande échelle (au-delà de la taille $\xi$ du blob) les différentes chaînes s'interpénètrent, s'ignorent, comme dans un fondu !

Que peut-on voir par DNPA ? Si le solvant est deutérié, et aucune des chaines ne l'est, on mesure les corrélations de fluctuations de concentration en polymère (courbe rouge), correspondant au signal «total », $\mathrm{S}_{\mathrm{T}}(\mathrm{q})$. Aux grands qs, qui donnent accès à l'intérieur du blob, cela revient à mesurer la conformation de la chaîne. Aux petits qqs, cela revient à mesurer la compressibilité osmotique de la solution, qui gouverne les fluctuations. On tend à $q->0$ vers une quantité proportionnelle à l'inverse de la compressibilité.

Mais on peut aussi marquer certaines chaines seulement. On peut alors obtenir, comme décrit en Figure 11, le signal intra chaîne $S_{1}(q)$ (qui donne le facteur de forme $P(q)$ de la chaîne après normalisation à 1 pour $q>0) . S_{1}$ passe de $q^{-3 / 5}$ aux grands $q$ à $q^{-2}$ aux petits $q(q$ $<1 / \xi)$, ce qui correspond à une chaîne gaussienne de blobs. Le facteur de forme dépend de la concentration. On voit que $S_{\mathbb{T}}(q)$ est inférieur a $S_{1}(q)$ aux $q<1 / \xi$, ce qui traduit l'écrantage des fluctuations de concentration par l'interpénétration. A partir de $S_{1}(q)$ et de $S_{T}(q)$, on peut obtenir une fonction de corrélation de paire distincte $\left(S_{2}(q)=S_{T}(q)-S_{1}(q)\right)$, qui est donc négative à $q<1 / \xi$. Mais notons qu'on ne peut pas définir dans cette situation de facteur de structure $S(q)=S_{\mathrm{T}}(q) / S_{1}(q)$, à la différence des suspensions de particules centrosymétriques, parce que les chaînes s'adaptent l'une à l'autre dans le processus d'interpénétration.. 

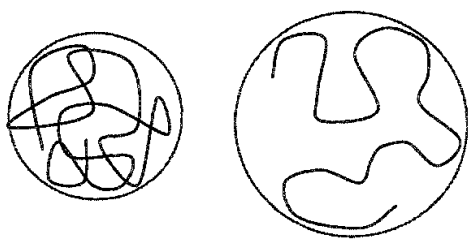

Gaussien

$\rightarrow$

\section{Volume exclus}

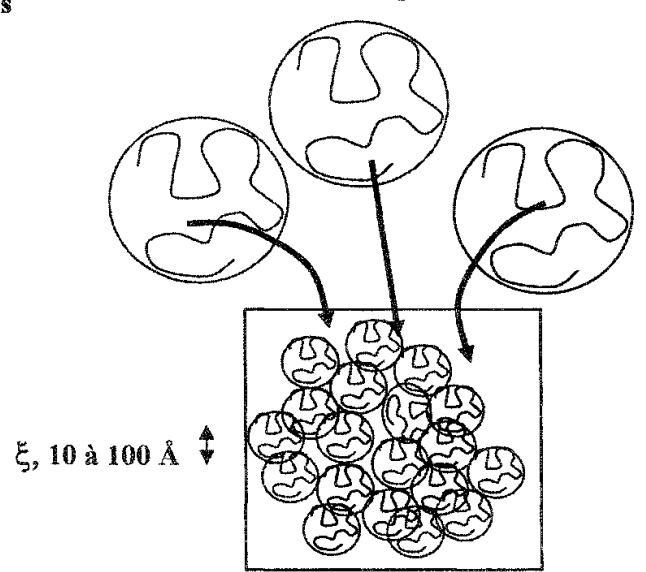

$-\log \left(-S_{\mathbf{Y}}(q)\right)$, « corrélation distincte »

Figure 11 : Solution semi-diluée, interpénétration, blob,et cross-over.

\section{2 Méthode de contraste moyen nul}

Un des intérêts du marquage par deutériation est de pouvoir observer une seule chaîne au milieu des autres, bien que toutes les chaînes soient interpénétrées entre elles. Pour cela on peut :

- réaliser plusieurs solutions à pourcentage de chaines deutériées variable, (la concentration totale deutériées + non deutériées restant constante), et extrapoler à concentration en chaînes deutériées nulle.

- ou bien utiliser un solvant mélange de solvant normal et de solvant deutérié, tel que la densité de longueur de diffusion soit intermédiaire entre celle du polymère hydrogéné et celle du polymère deutérié. La Figure 12 montre que dans ce cas l'ensemble des 4 contributions inter chaînes distinctes est nulle. L'intensité ne contient donc plus que les termes intra-chaîne, proportionneis à $S_{1}(q)[1,5]$. 


\section{Extrapolation à $\phi_{\mathrm{D}} \rightarrow 0$ काotal constant}

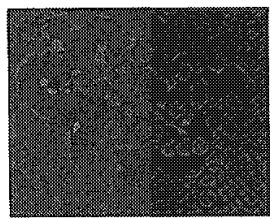

\section{Solvant tel que $K_{i}=0$}

$$
\mathbb{I}(q)=\phi_{D} S_{1}+\phi_{D}^{2} S_{2}
$$

\section{Contraste moyen nul}

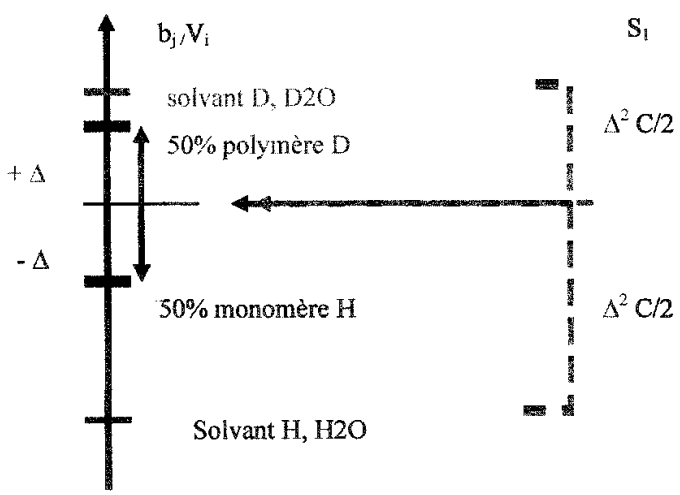

$\mathrm{S}_{2}$

$\mathrm{DD}(+\Delta)(+\Delta)$

$D H(+\Delta) \cdot(-\Delta)$

$H D(-\Delta)(+\Delta)$

$H H(-\Delta) \cdot(-\Delta)$

$=0$

Figure 12 : Solution semi-diluée ; mesure de $S_{1}$ par contraste moyen nul

\section{3 Dynamique des chaînes en solution semi-diluée}

On retrouve dans la dynamique des comportements différents dans les mêmes différents domaines de $q$ que pour la statique [8]. En Figure 13, on voit que pour $q>1 / \xi$, à la conformation de volume exclus correspond une dynamique de Zimm, comme en solution diluée, tant pour $S_{T}(q, t)$ que pour $S_{1}(q, t)$, facteur dynamique intra-chaîne,mesuré pour la chaîne seule au milieu des autres chaînes grâce à la mesure par Contraste Moyen Nal appliquée à l'écho de spin [8]). Pour $q<1 / \xi$, à la conformation gaussienne (volume exclus écranté) vue pour $S_{1}(q)$ correspond une dynamique de Rouse ("backflow" écranté) pour $S_{1}(q, t)$. Pour les fluctuations totales de concentration en polymère dans le solvant (facteur $S_{T}(q, t)$ ), on observe les fluctuations osmotiques, variant comme $\exp \left(-D_{\text {coop }} q^{2} t\right)$, où $D_{\text {coop }}$ est un coefficient de diffusion « coopératif »; $\Gamma / \mathrm{q}^{2}$ est donc constant . 
Dilué
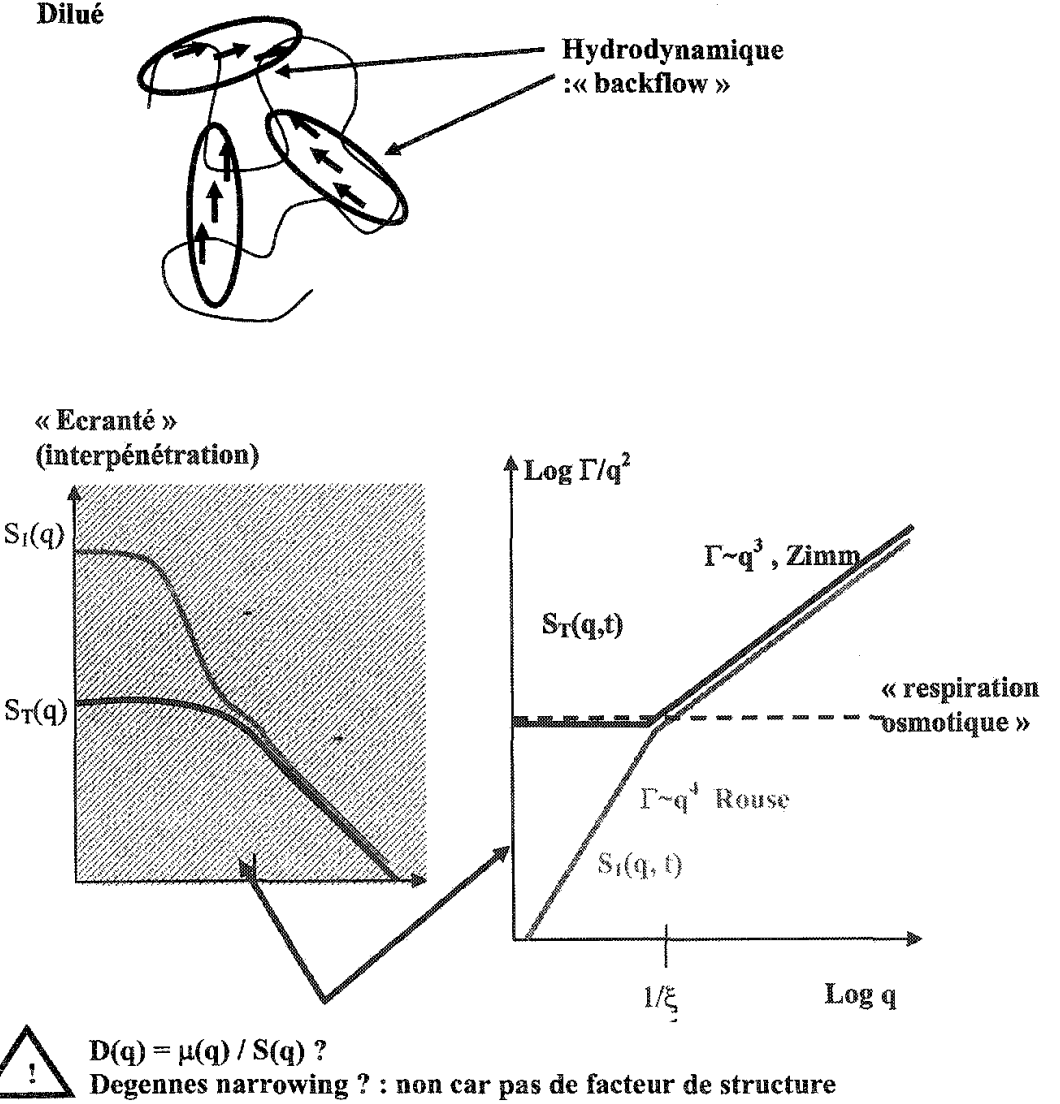

Tigure 13 : Solution semi-diluée: comparaison statique (courbe de gauche versus q) et dynamique(variation de $\Gamma / \mathrm{q}^{2}$, à droite) $[8]$.

\section{FONDUS DE CHAINES}

\subsection{Lffets stériques: trou de corrélation}

Dans un liquide de chaines, il n'y a pas d'effet de volume exclus sur la conformation, car chaque chaîne rencontre indifféremment une autre partie d'elle-même ou une autre chaîne. Pourtant, le volume non nul des monomères mène à un effet frappant lorsque toutes les chaînes sont marquées sur les mêmes séquences de monomère (e.g. toutes les chaines marquées au bout). On observe un maximum dans le signal (Figure 14) aux environs de $q=$ $\mathbb{1} \mathbb{R}_{\mathrm{g}}[2]$. En mots simples, cela vient du fait que la taille maximum des amas de monomères marqués est limiée par le nombre de chaînes qæe l'on peut placer dans un même volume 
donné. Il y a donc une sorte de répulsion des chaînes supplémentaires (« trou de corrélation " [2]).

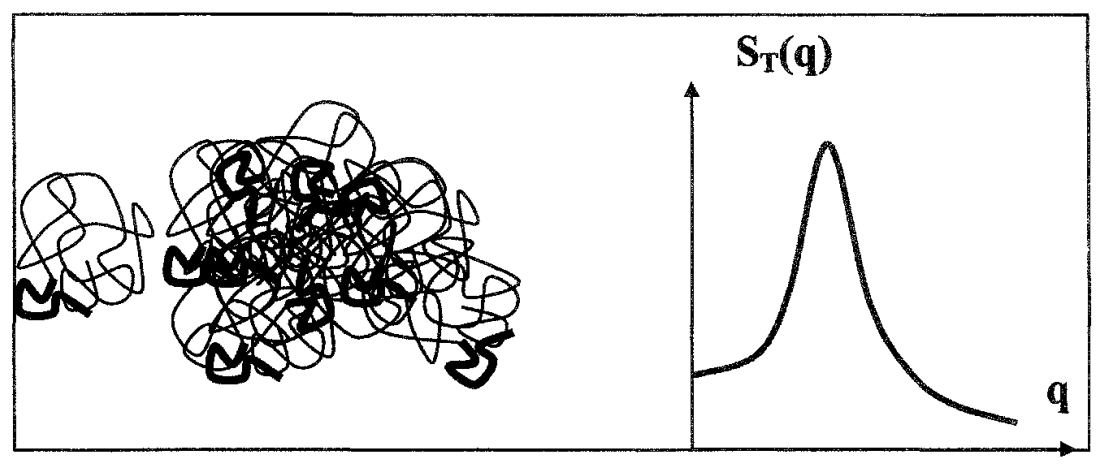

Figure 14: Fondu de chaînes toutes marquées à l'identique : un pic inattendu (lié dans l'espace direct, au « trou de corrélation " [2].

\subsection{Effets « topologiques » ? Chaînes « enchevêtrées » : dynamique de reptation}

Ces effets se retrouvent, $d$ 'un certain point de vue, sur la dynamique. Au modèle de la chaîne de Gauss correspond la dynamique de Rouse, à condition que la chaîne soit libre dans l'espace. Cette dynamique est observée jusqu'à des échelles en fait surprenamment grandes (plus de 50 Angströms). Cependant au dessus de cette taille, la chaîne semble bloquée, piégée, par les autres chaînes. Par écho de spin, on observe que la fonction $\mathrm{S}(\mathrm{q}, \mathfrak{t})$ ne relaxe pas jusqu'à zéro aux temps grands, mais présente un plateau (Figure 15, haut). La dépendance de ce plateau avec q correspond au mouvement de Rouse d'une chaîne confinée dans un puits de potentiel harmonique autour de la position moyenne de l'axe de la chaine, c'est-à-dire dans une sorte de « tube » de diamètre $\mathrm{D}$. On trouve pour $\mathrm{D}$ une valeur en accord avec le nombre de monomères entre deux enchevêtrements, $N_{e} . N_{e}$ est estimé à partir du module d'un fondu de grandes chaines dans le régime de temps dit "plateau caoutchoutique ». Dans ce régime, un fondu de grandes chaînes se comporte comme un caoutchouc élastique (avant de couler -on dit « fluer 》sur des échelles de temps plus grandes). $N_{e}$ est l'équivalent du nombre de monomères entre deux points de réticulation dans un caoutchouc réticulé chimiquement (qui ne flue pas). La dynamique de la chaîne dans ce tube a été décrite par Edwards, de Gennes [2], et Dơ̈ [3], par le modèle dit de reptation. Ceci est schématisé par les dessins de la Figure 15, où l'on voit comment la chaîne passe d'un mouvement de Rouse aux temps courts, à un mouvement au sein de son tube. La chaîne renouvelle alors son «tube» par les extrémités seulement, en un mouvement de navette. 


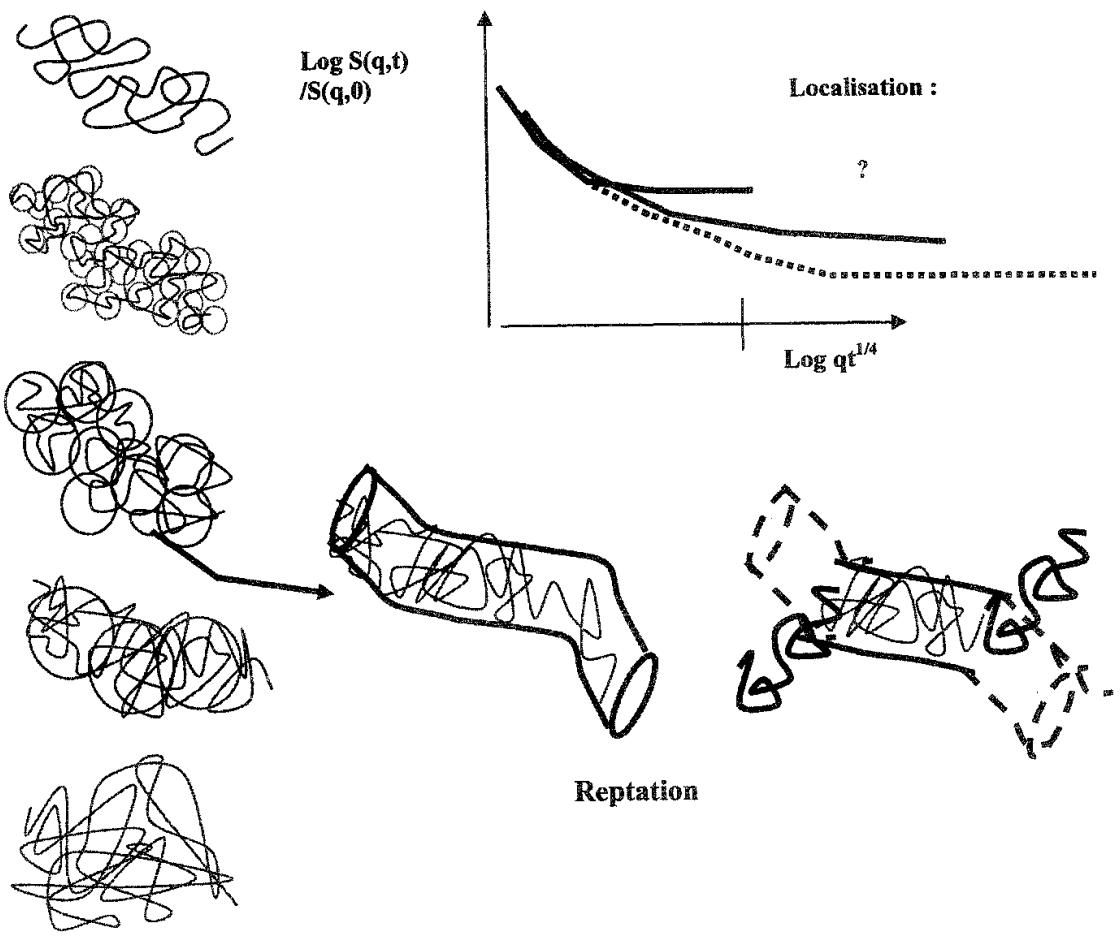

Figure 15 : Dynanique de reptation dans un tube formé par les autres chaînes.

La taille du tube correspond à celle d'une chaîne de $\mathrm{N}_{\mathrm{e}}$ monomères, soit plusieurs dizaines, voire plusieurs centaines (180 pour le polystyrène).

Ces effets dits $d$ ' "enchevêtrements " sont mal expliqués; notons que les données neutrons sur les rayons de giration, et la longueur de persistance permettent de proposer des modèles de plus en plus précis. En particulier, plus les chaînes sont rigides ( $\mathbb{L}_{p}$ grand) et minces (diamdtre axial petit), plus elles se bloquent les unes les autres (comme un ensemble d'aiguilles), plus les distances entre enchevêtrements sont courtes (Figure 16). Les valeurs précises de tailles données par les mesures de DNPA peuvent être très utiles pour progresser sur ce sujet. 


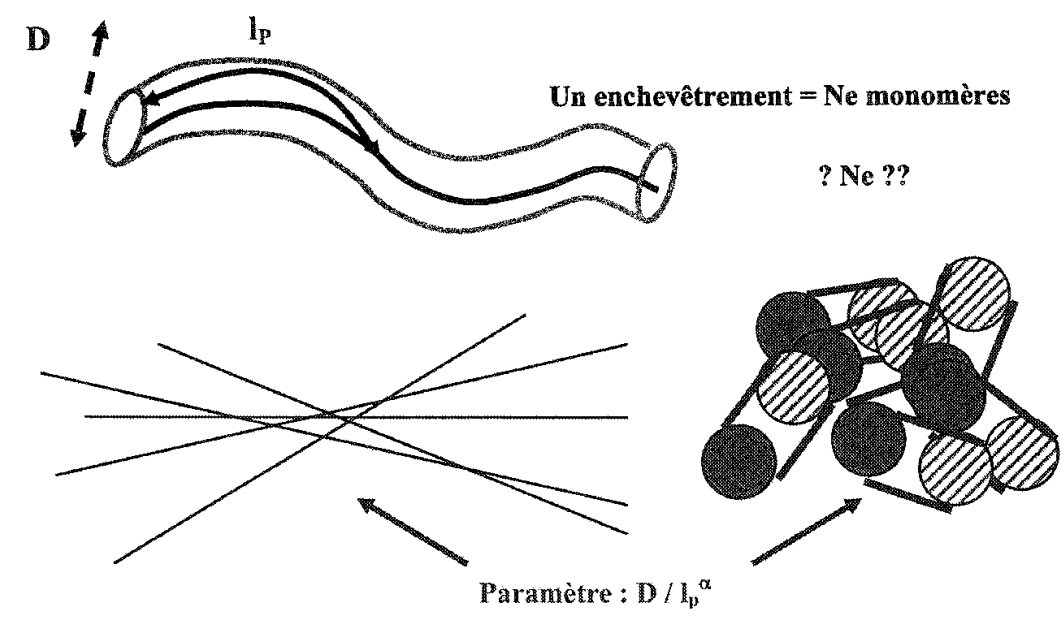

Lin : $N_{e}=400 D^{4} / b_{c} l_{P}^{3}$

Fetters et al. : dans un volume, une autre chaîne

: En solution semi-diluée, un enchevêtrement $=$ Ne blobs !!!

Même Ne qu 'en fondu !

Figure 16 : Quelle origine pour les enchevêtrements ?

4.3 Retour à l'isotrope après une marche de déformation : visualisation du tube

Il est possible d'observer dynamique de Rouse et de reptation pendant le retour à la configuration isotrope après une déformation brusque [9] (Figures 17 et 18). Le signal anisotrope doit être enregistré sur un détecteur bidimensionnel XY. Le long de chaque direction, l'intensité présente un « cross-over », en fonction de l'échelle d'observation, entre une déformation homothétique (" affine ») à la déformation macroscopique - à grande échelle (petit q), et une conformation quasi isotrope à très petite échelle (grand q), qui traduit la capacité de la chaîne à conserver le maximum d'entropie conformationnelle: cela est à l'origine même de l'élasticité caourchoutique. La valeur de q de cross-over devrait est reliée au modèle (voir texte en bas de la Figure 18). 


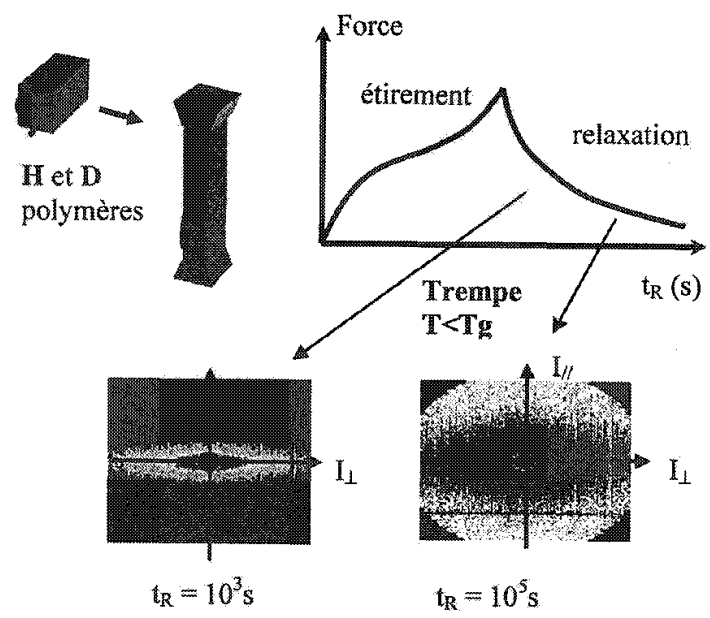

Figure 17 (ci-dessus) : Observation du facteur de forme d'une chaîne dans un fondu pendant une relaxation après une déformation rapide [9].

Figure 18 (ci-dessous) : Retour vers l'équilibre du facteur de forme suivant le modèle de Rouse puis de reptation [9].

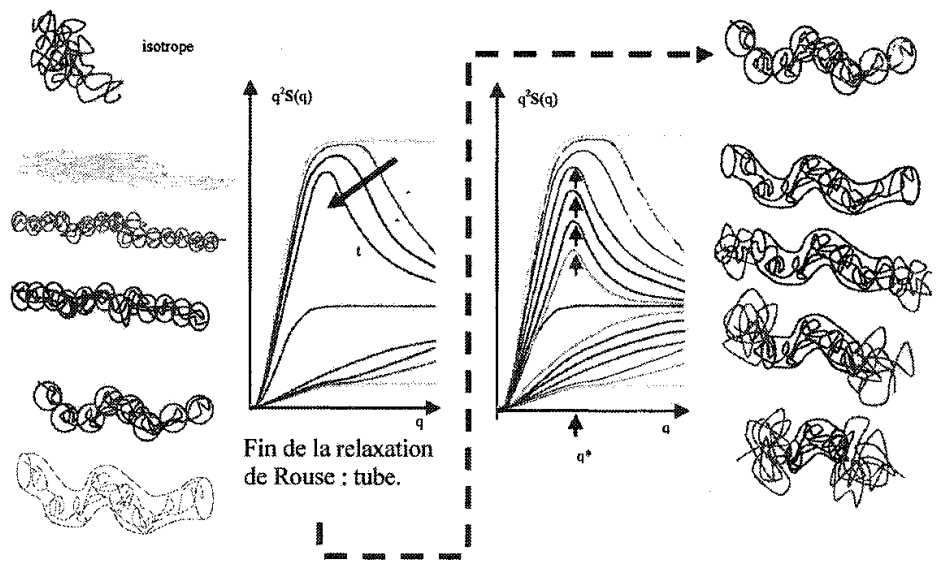

Modèle de Rouse.

Longueur seuil de perte d'affinité $\sim t^{1 / 4}$. q au "cross-over" $" q^{*} \sim t^{-1 / 4}$.

\section{Modèle de reptation}

Taille perte $d^{\text {'affinité }}=$ diamètre $d u$ tube $\Rightarrow q^{*}$ constant. La chaîne se désengage par les extrémités $\Rightarrow$ intensité $=$ addilion de partie centrale déformée + extrémités isotropes 


\section{AUTRES ARCHITECTURES, COPOLYMERES, POLYMERES CRISTAUX LIQUIDES}

La structure linéaire de base des chaînes permet de nombreuses variations spécifiques à ces matériaux. Nous les évoquons brièvement car cela nous entraînerait très loin !

D'une part on peut jouer sur la nature des monomères. Le greffon peut avoir une tendance intrinsèque à l'auto-organisation, qui se répercutera sur le polymère. C'est le cas des polymères cristaux liquides qui, grâce à leurs monomères «nématogènes 》, présentent des phases nématique et smectique. On peut caractériser le facteur de forme de telles chaînes dans les phases correspondantes (exemple de la phase nématique en haut de la Figure 19) [10]. La susceptibilité à une déformation mécanique est importante. Les régimes de relaxation après élongation comme la reptation sont profondément modifiés par l'état cristal liquide [11]. Une phase nématique différente, induite par le cisaillement, a été observée par rhéo-DNPA [12] (bas de la Figure 19).

Diffusion aux Petits angles (facteur de forme)
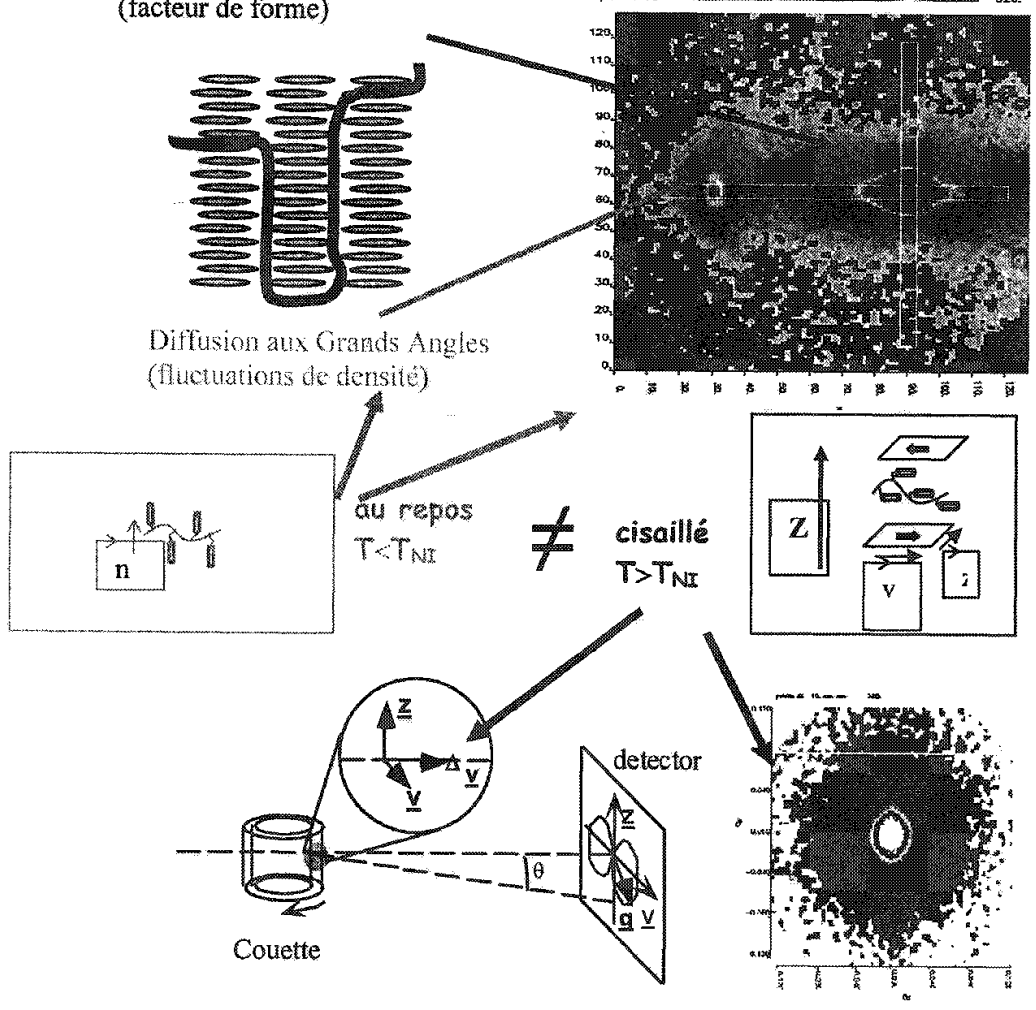

Figure 19: Polymères cristaux liquides : observation d'une chaine en phase smectique orientée par un champ magnétique (en haut, [9]) et dans une phase smectique induite par le cisailiement (en bas, [11]), montrant unie orientation squelette/nématogènes différente. 
Par ailleurs, en mélangeant plusieurs espèces de monomères, on fabrique des copolymères [1,2], statistiques ou à blocs (AAAAAA....-BBBBBBB par ex.) dont le diagramme sera voisin de celui des molécules amphiphiles. Ces mélanges permettent de nombreuses combinaisons de propriétés physiques $\left(T_{g}\right.$, propriétés mécaniques) des deux constituants (poly-A et poly-B), mais celles-ci sont aussi liées à la géométrie des phases.

Enfin, avec un monomère simple donné, on peut créer des architectures branchées [1, 2] : depuis les étoiles de branches partant d'un centre, qui présentent à l'état individuel un facteur de forme intéressant si l'on tient compte de la concentration variable lorsque l'on s'éloigne du cour (modèle de Daoud - Cotton), en passant par les polymères à nombreux points de branchement (hyperbranchés) on arrive aux polymères en peigne comportant des greffons branchés latéralement. Toutes ces formes, et les structures générées par leur interpénétration mènent à une variété de structures inter-chaîne, et d'enchevêtrement d'une grande richesse que les techniques de neutron peuvent explorer en détail grâce au marquage.

\section{RETICULATS DE CHAINES : CAOUTCHOUCS ET GELS}

Lorsque les chaînes polymères sont liées entre elles par des liaisons chimiques ou physiques (Figure 20 en haut à gauche), elles forment un réseau tridimensionnel, dont la structure est rarement aussi simple que celle représentée en Figure 20 en haut. Plusieurs possibilités de marquage donnent accès à plusieurs informations.

\subsection{Naissance du réseau : amas fractals et point de gel}

On peut étudier l'agrégation progressive des chaînes (formation de morceaux de réseau, ou prégel) entre elles en présence de solvant si ce dernier est deutérié. Ce processus peut révéler une première structure due au processus même de réticulation. $\mathrm{Si}$ ce processus est suffisamment aléatoire, le signal peut rester voisin de celui d'avant la réticulation. Dans ce cas on n'observera les caractéristiques des amas qu'en ajoutant du solvant : ceci aura pour effet de séparer, de désinterpénétrer les amas (comme les chaînes pour la dilution d'une solution semidiluée), et ainsi de révéler, par exemple une structure fractale des amas, dont la dimension apparente $d_{f}$ peut varier entre 1.6 et 2.5 [2]. La taille du plus grand de ces amas croit avec le progrès de la réticulation, jusqu'à devenir infinie au point de gel. A de plus grands taux de réticulation on complète, au contraire les nouds manquants pour aboutir à une structure qu'on s'attend à devenir plus régulière. Nous allons voir que ce n'est pas tout à fait le cas.

\subsection{Réseau terminé : quelle structure?}

Pour un gel «terminé ", en présence de solvant (deutérié), le réseau à l'état de préparation peut révéler une première structure due au processus même de réticulation. Mais comme pour avant le point de gel ( prégel »), le signal peut rester voisin de celui d'avant la réticulation si celle-ci est suffisamment aléatoire. Dans ce cas il sera révélateur comme pour un prégel, d'ajouter du solvant: ceci provoquera un gonflement du gel (déformation isotrope). Si le solvant est marqué, le signal dû aux fluctuations de concentration, montrera souvent que les fluctuations sont amplifiées par le gonflement. Elles reflètent le plus souvent l'existence de fluctuations gelées (piégées par la réaction) de la répartition spatiale des points de réticulation [13].

On pourra aussi marquer (généralement avant réticulation) certaines des chaînes à partir desquelles le réseau est construit. S'il est issu de la réticulation de courtes chaînes par les bouts, on pourra utiliser une fraction de chaines marquées, et aboutir à un réseau à « mailles marquées \%. Si la réticulation est faite sur des chaines plus longues par plusieurs ponts par 
chaîne, l'utilisation d'une fraction de chaînes deutériées mènera à des « chemins marqués ". Ces objets sont souvent peu modifiés par la réticulation. C'est une déformation soit par gonflement ou séchage, soit par action mécanique (e.g. étirement) qui révèlera des effets souvent surprenants dus aux différentes structures du réseau.

Un cas analogue est l'effet de l'étirement sur ce facteur de forme pour le réticulat en masse, sans solvant (qui sera un " caoutchouc " au-dessus de $T_{g}$ ). En fait le comportement est voisin de celui d'une chaîne marquée étirée dans un fondu de chaîne. Une grande analogie est observée dans le régime transitoire de plateau caoutchoutique correspondant au début de la reptation, avec la déformation dans un caoutchouc permanent. Le long de chaque direction, l'intensité présente un "cross-over », en fonction de l'échelle d'observation, qui traduit la capacité de la chaîne à conserver le maximum d'entropie conformationnelle : cela est à l'origine même de l'élasticité caoutchoutique. $\mathrm{La}$ valeur de $q$ de cross-over entre une déformation homothétique (" affine ") à grande échelle (petit q), et une conformation quasi isotrope à grand $\mathrm{q}$ devrait être reliée au taux de réticulation: on observe cependant des comportements anormaux fréquents qui montrent que la déformation n'est pas affine. Ils peuvent s'expliquer par les hétérogéneités de réticulation.

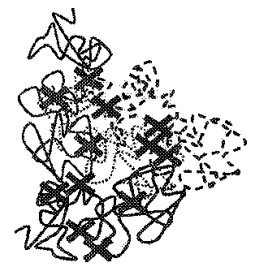

Fabrication

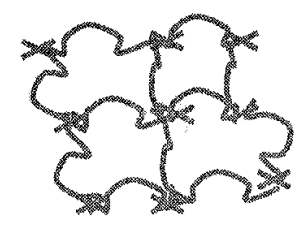

Image simpliste

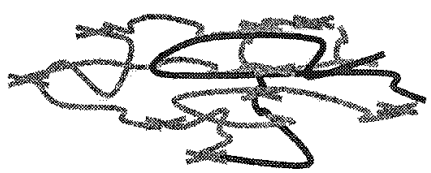

Chemin marqué étiré

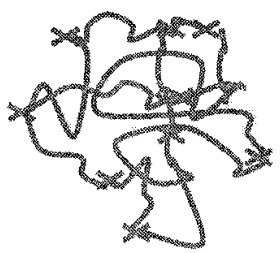

Etat réel

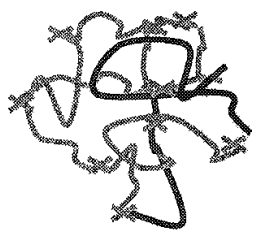

Chemin marqué

Figure 20 : Réticulats de chaînes dans les cacutchoucs et les gels. 


\title{
7. UN AUTRE EXEMPLE DE RHEO-DNPA / SOLUTION SEMUDILUEE SOUS CISAILLEMENT
}

Nous allons prendre un deuxième exemple de déformation d'un système polymère, provoquée cette fois par un écoulement : on peut parler alors de « rhéo-DNPA ». On cisaille dans un viscosimètre de Couette (deux cylindres coaxiaux avec un entrefer de l'ordre d' $1 \mathrm{~mm}$ ) une solution classique de polymère en solvant organique. Ce type de solution présente généralement un diagramme de phase température - concentration (Figure 21), qui sépare le domaine de température en trois zones : bon solvant, où la chaîne est parfaitement soluble, solvant thêta où la chaîne a la même énergie libre d'interaction avec le solvant qu'avec ellemême, et mauvais solvant [14].

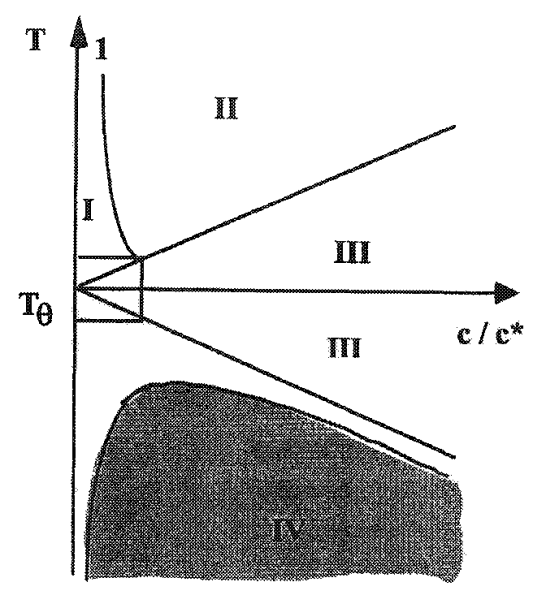

\author{
I Bon solvant, dilué
}

II Bon solvant, semi-dilué

III solvant neutre (《theta »)

IV Mauvais solvant (séparation liquide - liquide)

Figure 21 : Diagramme température -concentration (Daoud - Jannink) pour une solution de polymères neutres en solvant organique.

Sous l'effet d'une déformation, des fluctuations géantes, anisotropes, apparaissent. On peut en tracer l'émergence par DNPA, jusqu'à ce que leur taille maximum mène à une forte diffusion anisotrope de la lumière; on peut même « raccorder » les deux mesures (Figure 23). II a été montré [15] que ces fluctuations sont dues d'abord au couplage entre effets mécaniques et fluctuations de concentration, amplifié à son tour par la proximité de la zone de mauvais solvant. Ces effets n'ont des conséquences rhéologiques (frappantes) qu'aux plus grands taux de cisaillement. 

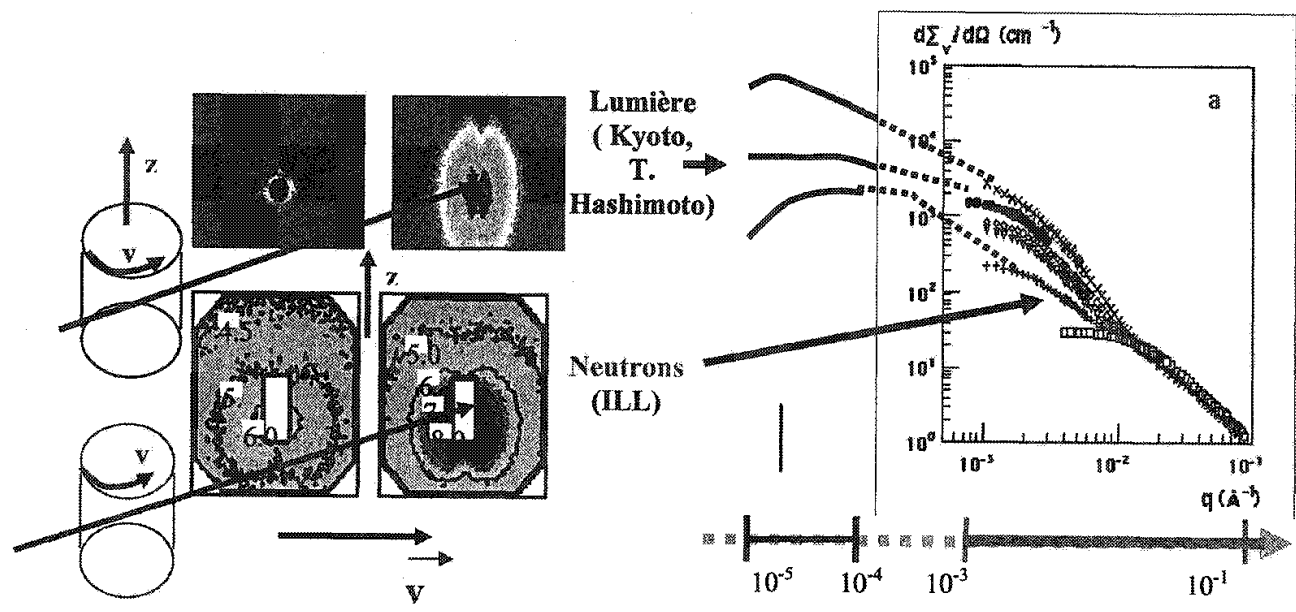

Espace direct : interfaces plus ou moins netfes
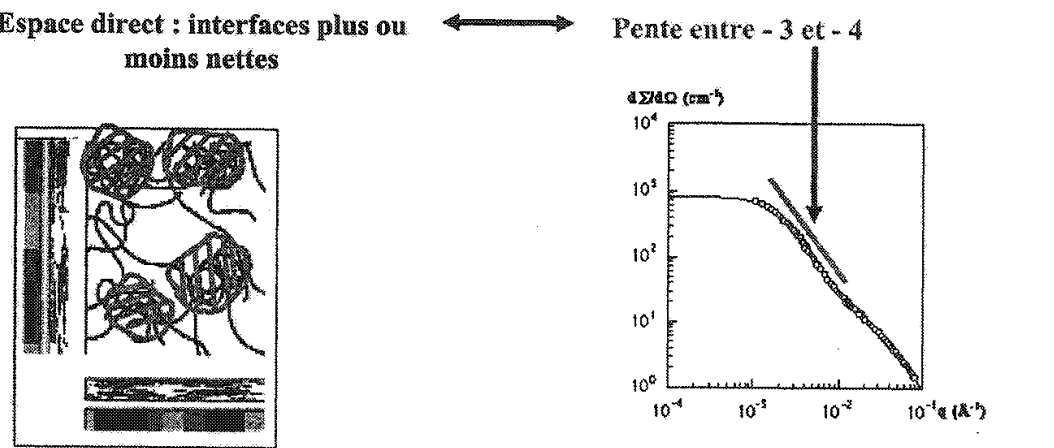

Figure 22 : Solution semi-diluée : structures induites par le cisaillement. [15]

\section{MELANGES TERNAIRES / VARIATION DE CONTRASTE}

Les polymères sont souvent associés à d'autres espèces (particules sur lesquelles ils sont adsorbés ou greffés, tensioactifs auxquels ils s'associent) dans la nature ou dans les formulations industrielles. Les complexes formés sont des systèmes multicomposants, pour lesquels la DNPA se révèle très utile grâce aux possibilités de marquage par deutériation $[1$, 4]. Prenons l'exemple d'un système ternaire de deux espèces 1 et 2 , et d'un solvant (noté S). De façon analogue au cas du mélange binaire (cf. ci-dessus), l'incompressibilité s'écrit:

$V_{1} n_{1}(r)+V_{2} n_{2}(r)+V_{S} n_{S}(r)=c s t e$

Ce qui permet de réécrire l'intensité :

$I=b_{12} n_{1}(q) n_{1}(-q)+2 b_{1} b_{2} n_{1}(q) n_{2}(-q)+b_{22} n_{2}(q) n_{2}(-q)$

+2 (detto pour $\left.n_{1}, n_{3}\right)+2$ (detto pour $\left.n_{2}, n_{3}\right)+$ (detto pour $n_{S}, n_{S}$ ) 


$$
\begin{aligned}
= & \left(b_{1}-b_{s} V_{1} / V_{S}\right) 2 S_{11}+2\left(b_{1}-b_{s} V_{1} / V_{S}\right)\left(b_{2}-b_{s} V_{1} / V_{S}\right) S_{12} \\
& +\left(b 2-b_{s} V_{1} / V_{S}\right) 2 S_{22}+\ldots \\
= & K_{1}^{2} S_{11}+K_{2}^{2} S_{2}^{2}+K_{3}^{2} S_{33} \\
& +2 K_{1} K_{2} S_{12}+2 K_{1} K_{3} S_{13}+2 K_{2} K_{3} S_{23}
\end{aligned}
$$

On voit - comme illustré en Figure 23 - qu'on peut annuler le contraste d'une des espèces par rapport au solvant. Pour cela on mélange solvant deutérié et non deutérié dans une proportion telle que le mélange a la même valeur de densité de longueur de diffusion que soit la particule, soit la chaîne. Ceci peut être fait par calcul, ou expérimentalement si on peut disperser séparément particules seules et chaînes seules dans ce même solvant (on détermine alors la fraction qui annule le signal du mélange binaire. $S i K_{1}$ ou $K_{2}$ est nul, on peut alors isoler la contribution $S_{11}$ ou $S_{22}$; avec un troisième mélange de solvant, connaissant $S_{11}, S_{22}$, $\mathrm{K}_{1}, \mathrm{~K}_{2}$, on déduira $S_{12}$ de l'intensité.
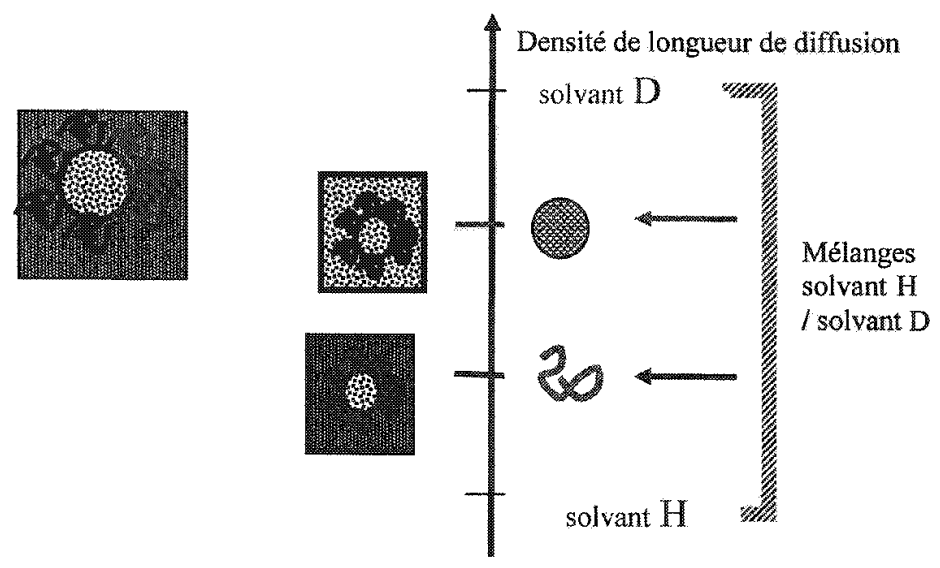

Figure 23 : Variation de contraste

\section{INTERFACES}

\subsection{DNPA sur des interfaces : principe.}

La DNPA permet d'accéder à la structure d'interfaces entre objets de petite taille, par exemple entre des sphères d'une espèce dispersée dans un solvant ou une matrice, et l'espèce hôte. Si l'interface est parfaitement lisse, on observe un signal dit de Porod en $1 / \mathrm{q}^{4}$, simplement proportionnel à la quantité de surface (surface spécifique) [4]. Si l'interface est moins nette, plus diffuse, comme pour une interpénétration des deux milieux, ce signal en $1 / \mathrm{q}^{4}$ peut être multiplié par la transformée de Fourier du profil de l'interface (Figure 24). Si la surface possède un profil fractal de dimension $d_{S}$, une loi en $1 / q^{6-d}$ peut être observée, ce qui donne accès à ds [16]. 


\section{Porod (1.q) multiplié}
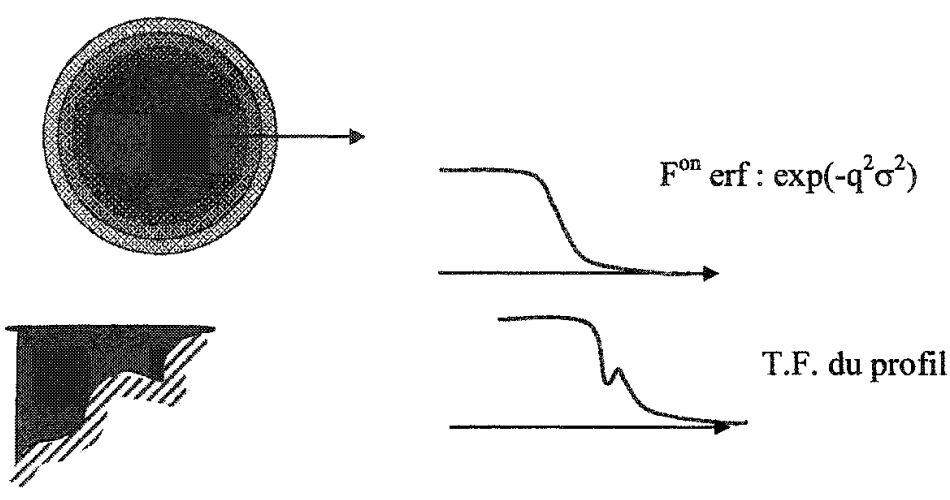

Fractal de surface $: 1 / q^{6-d S}$

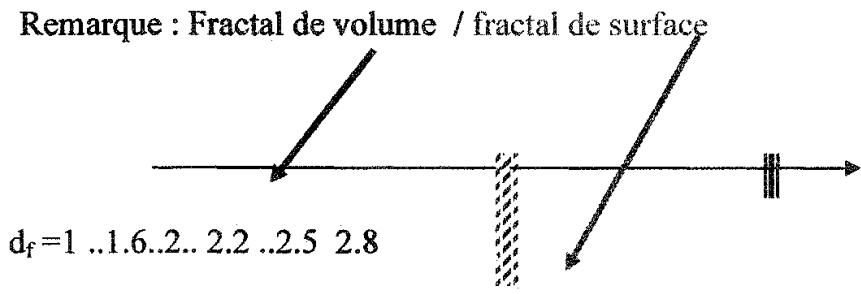

$$
2.8 .3 \ldots 3.5 \ldots \quad 4
$$

Figure 24: schémas d'une interface.

9. 2 Etude d'une interface par réflectivité de neutrons : cas de l'interpénétration de deux polymères

S'il s'agit de surfaces planes macroscopiques, la technique de réflectivité de neutrons est bien adaptée à l'étude d'interfaces: liquide - liquide, liquide - air, liquide - substrat, qui provoqueront souvent des structures interfaciales spécifiques aux polymères qui viennent s'y adsorber (brosse auto-similaire, brosse dense [2]). Une autre catégorie de situation est produite par la technique de spin coating qui permet de déposer des couches minces planes de polymère (à l'état vitreux généralement). Nous prendrons un exemple dans cette demière catégorie. La Figure 25 illustre le cas [17] d'une couche de polystyrène réticulé déposée sur une couche de polystyrène non réticulé, à l'état fondu. En chauffant, on passe au dessus de $\mathrm{Tg}$ et les chaînes peuvent diffuser.

La courbe de réflectivité $R(k)$ - $k$ est l'équivalent de q- montre des oscillations liées à l'épaisseur des deux couches ; lorsque l'interface s'élargit, ces oscillations s'estompent. Cela donne un bon critère pour ajuster aux données un modele de profil d'interface de type fonction erreur, caractérisée par une largeur à mi hauteur $\sigma$. Cette largeur correspond au parcours de diffusion moyen des chaînes dans ce temps t. Des modèles plus sophistiqués simulant la progression stochastique des chaines dans un gradient de concentration donnent de 
bons résultats [18]. On voit en Figure 26 que l'épaisseur de pénétration $\sigma$ croit continûment pour des chaînes libres petites (que le réseau accepte dans tout son volume) alors que des chaînes libres grandes ne pénètrent qu'en surface : $\sigma$ se stabilise dans ce cas à une valeur constante. Cette capacité de déformation du réseau à accueillir les chaines s'exprime en fonction du rapport nombre de monomères moyen dans une chaîne mobile / nombre de monomères moyen entre points de réticulation $\mathrm{N}_{\text {mobile }} \mathrm{N}_{\mathrm{c}}$ semble bien être le paramètre contrôlant I'ensemble des résultats.
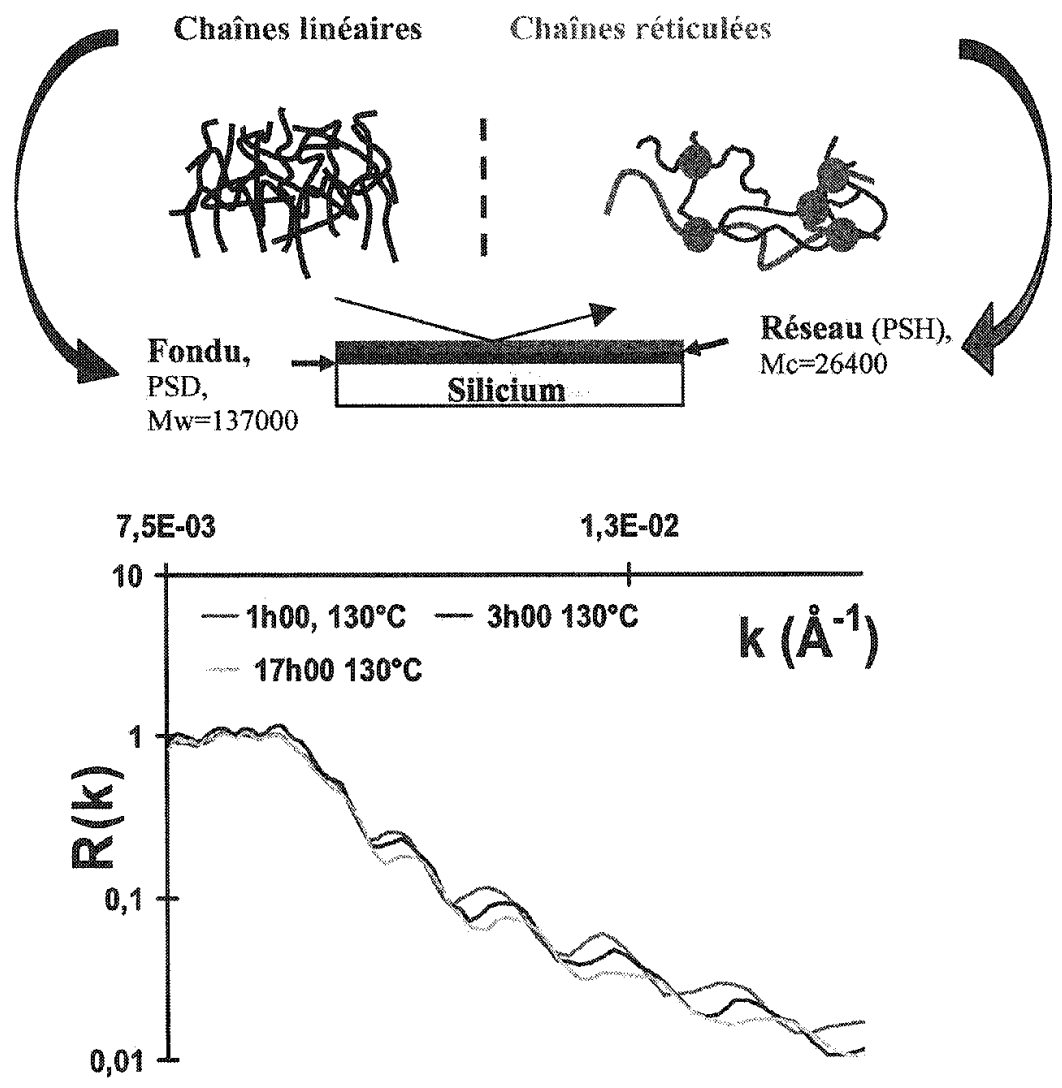

Figure 25: étude par réflectivité [17] de l'évolution au recuit de l'interface entre deux couches de polymère, l'une réticulée, l'autre pas. 

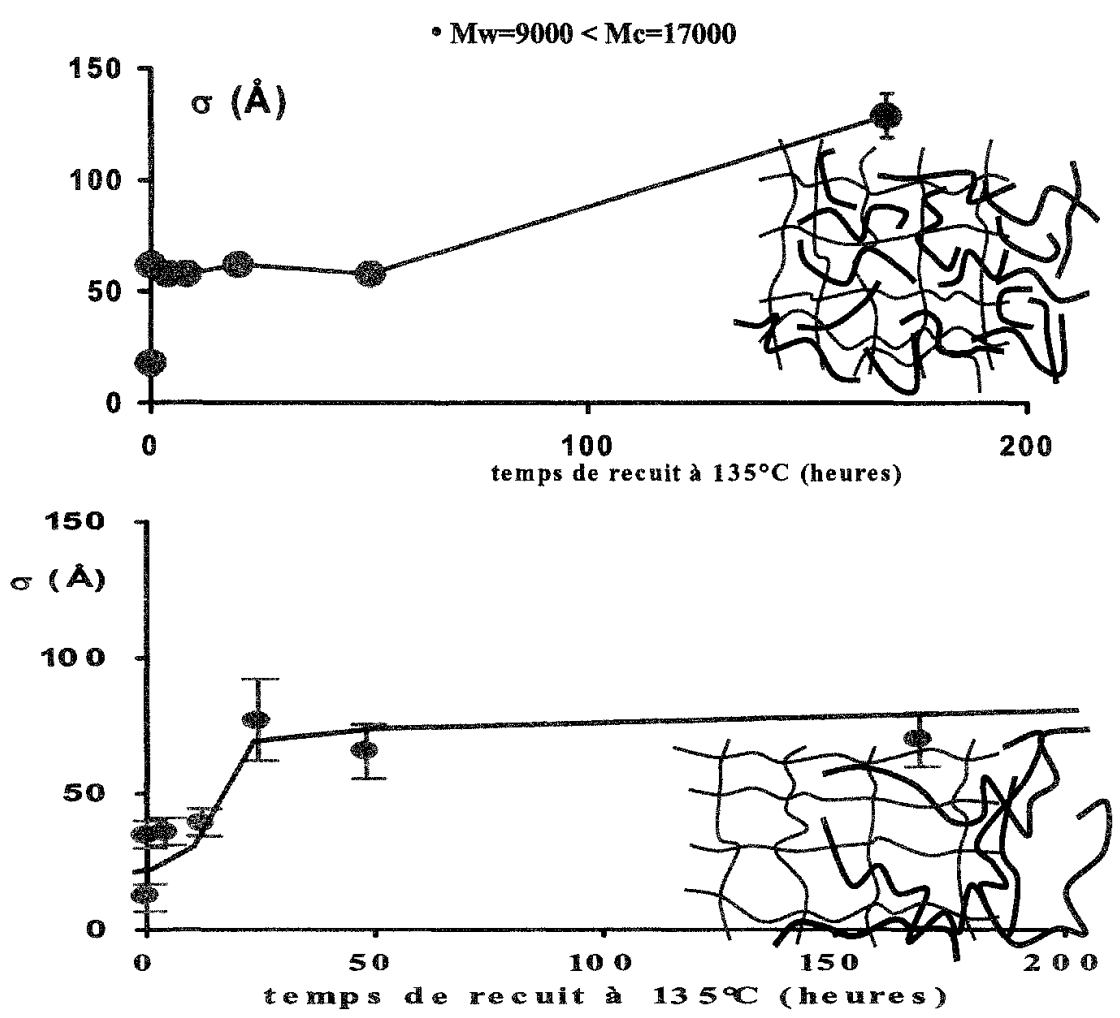

Figure 26: variation de l'épaisseur de l'interface $\sigma$ avec la durée du recuit : en haut pour de petites chaînes pénétrant complètement dans le réseau, en bas pour des chaînes plus grandes que la maille, qui ne gonflent qu'une interface [17].

\section{CONCLUSION}

Bien que matériaux désordonnés, les polymères présentent des géométries élégantes qui peuvent être décrites complètement mathématiquement. La capacité de l'objet élémentaire, la chaîne, à explorer de grandes échelles, mène donc à des conformations originales, qui de par leur faible compacité permettent des arrangements non moins originaux. Cette grande gamme de tailles privilégie les techniques de neutron aux petits angles, DNPA, réflectivité, Echo de spin, comme méthodes d'êtude. Une des richesses en est l'observation de changement de comportement avec le vecteur q. Ces structures mènent aussi à des dynamiques originales dans leur dépendance en $q$ et en fréquence. En dehors de l'étude per se de ces matériaux aux grandes possibilites d'application (avec beaucoup de produits nouveaux à venir), il est probable, et souhaitable, que les concepts qui en ont été extraits s'avèrent utiles à la description d'autres matériaux amorphes. 


\section{Références}

1. H. Benoit, J.S. Higgins, Polymer and neutron scattering, Oxford (1994).

2. P.G. de Gennes, Scaling concepts in polymer physics, Cornell University Press, Ithaca (1979).

3. M. Doï, S.F. Edwards, The Theory of polymer dynamics, Clarendon Press (1988).

4. J.P. Cotton Ed, Diffusion de neutrons aux Petits Angles, Ecole des Journées RossatMignod, Albé , Editions de Physique EDP (1999).

5. M.N. Spiteri, F. Boue, A. Lapp, J.P. Cotton, Phys. Rev. Lett., 77, 26, 5218-5220 (1996).

6. B. Farago, A. Arbe, J. Colmenero, R. Faust, U. Buchenau, D. Richter, Phys. Rev .E (2002).

7. D.Richter, B. Ewen, B. Farago, T. Wagner, Physical Review Letters, 62 , 18 (1989).

8. T. Csiba, G. Jannink, D. Durand, R. Papoular, L. Auvray, F. Boué, J.P.Cotton, R. Borsali, J. Phys. II, 381-396 (1991).

9. F. Boué, Transient relaxation mechanisms in elongated melts and rubbers investigated by SANS, Adv. Pol. Sci. 82 (1987).

10. L. Noirez, P. Keller, J.P. Cotton, Liq. Cryst. 18, 129 (1995).

11. V. Fourmaux-Demange, A. Brûlet, F. Boué, P. Davidson, P. Kelleri, J.P. Cotton, The European Physical Journal E, 1, 301-317 (2000).

12. C. Pujolle, L. Noirez, Nature, 409, 167 (2001).

13. E. Mendes Jr, P.Lindner, M. Buzier, F. Boué, J.Bastide, Physical Review Letters, 12 , p1595-1598 (1991).

14. J.P. Cotton, M. Nierlich, F. Boué, M. Daoud, B. Farnoux, G. Jannink, R. Duplessix, C. Picot, J.Chem.Phys. 65, 1101 (1976).

15. I. Morfin, Thèse, Université Joseph Fourier, Grenoble, 2000, et I. Morfin, P. Lindner, F. Boué, Macromolecules, 32, 7208-7223 (1999).

16. S.K Sinha, E.B. Sirota, S. Garoff, H.B. Stanley, Phys. Rev. B, 38, 2297 (1988).

17. G. Bacri, Thèse, Université de Paris-Sud Orsay (2000).

18. T. Russ, R. Brenn, F. Abel, F. Boué and M. Geoghegan, The European Physical Journal E, 4, 419-433, (2001). 